\title{
Loops and spurs: The angular power spectrum of the Galactic synchrotron background
}

\author{
Philipp Mertsch ${ }^{a}$ and Subir Sarkar ${ }^{b, c}$ \\ ${ }^{a}$ Kavli Institute for Particle Astrophysics \& Cosmology, 2575 Sand Hill Road, M/S 29, Menlo \\ Park, CA 94025, USA \\ ${ }^{b}$ (On sabbatical leave from) Rudolf Peierls Centre for Theoretical Physics, University of \\ Oxford, 1 Keble Road, Oxford OX1 3NP, UK \\ ${ }^{c}$ (Visiting Professor at) Niels Bohr Institute, Copenhagen University, Blegdamsvej 17, 2100 \\ Copenhagen $\varnothing$, Denmark \\ E-mail: pmertsch@stanford.edu, sarkar@nbi.dk
}

\begin{abstract}
We present a new model of the diffuse Galactic synchrotron radiation, concentrating on its angular anisotropies. While previous studies have focussed on either the variation of the emissivity on large $(\sim \mathrm{kpc})$ scales, or on fluctuations due to MHD turbulence in the interstellar medium, we unify these approaches to match the angular power spectrum. We note that the usual turbulence cascade calculation ignores spatial correlations at the injection scale $(\sim 100 \mathrm{pc})$ due to compression of the interstellar medium by of $\mathcal{O}(1000)$ old supernova remnants - the 'radio loops' only four of which are visible in radio maps. This new component naturally provides the otherwise missing power on intermediate and small scales in the all-sky map at $408 \mathrm{MHz}$. Our model can enable more reliable subtraction of the synchrotron foreground for studies of CMB anisotropies or searches for dark matter annihilation. We conclude with some remarks on the relevance to modelling of the polarised foreground.
\end{abstract}

Keywords: Galactic radio emission, Cosmic microwave background, Interstellar medium turbulence, supernova remnants: radiative phase 


\section{Contents}

1 Introduction $\quad 1$

2 The observed angular power spectrum 5

3 Modelling of the angular power spectrum $\quad 6$

3.1 Definitions 6

$\begin{array}{lll}3.2 & \text { Large-scale variation of synchrotron emissivity } & 7\end{array}$

3.3 Small-scale variation of synchrotron emissivity 8

3.4 Free-free emission 11

$\begin{array}{ll}3.5 & \text { Unsubtracted point sources } \\ 3.6 & \text { Shell }\end{array}$

3.6 Shells of old SNRs 11

$\begin{array}{lll}\text { 3.6.1 Specific emissivity of a shell } & 12\end{array}$

$\begin{array}{ll}\text { 3.6.2 Line-of-sight integral over a shell } & 13\end{array}$

$\begin{array}{ll}\text { 3.6.3 The Galactic population of radio loops } & 14\end{array}$

4 Results $\quad \mathbf{1 5}$

4.1 APS from conventional model $\quad 15$

$\begin{array}{lll}4.2 & \text { APS adding SNR shells } & 16\end{array}$

$\begin{array}{llr}5 & \text { Discussion } & 18\end{array}$

6 Conclusions 20

$\begin{array}{llr}7 & \text { Acknowledgements } & 21\end{array}$

$\begin{array}{ll}\text { A The monopole compared to the total power } & 21\end{array}$

B Auxiliary function $J_{l}(a) \quad 22$

\section{Introduction}

Radio maps of the sky contain a wealth of information on non-thermal astrophysical processes. The sources of this radiation range from compact objects like pulsars with magnetospheres extending over hundreds of kilometers, to radio galaxies hundreds of kiloparsecs across, thus spanning over 16 orders of magnitude in size. The total brightness of the radio sky is dominated by diffuse emission from the Galaxy at frequencies between tens of $\mathrm{MHz}$ and a few $\mathrm{GHz}$ [1]. It is produced by synchrotron radiation of cosmic ray (CR) electrons and positrons ${ }^{1}$ gyrating in the Galactic magnetic field (GMF) and for typical field strengths of $\mathcal{O}(\mu \mathrm{G})$, the $\mathrm{CR}$ electron spectrum is probed between hundreds of $\mathrm{MeV}$ and tens of $\mathrm{GeV}$. The frequency and angular dependence of this diffuse emission encodes both the properties of the $\mathrm{CR}$ electron sources, e.g. their spectrum and spatial distribution, and of the GMF and its effect on the propagation of CRs. Therefore, studies of the diffuse radio background can in

\footnotetext{
${ }^{1}$ In the following, when using the term 'electrons' we refer to both electrons and positrons.
} 
principle determine crucial parameters of the propagation of CRs, e.g. the size of the (radio) halo in which they are confined.

The diffuse synchrotron emission at GHz frequencies also constitutes an important foreground for studies of cosmologically important angular anisotropies in the cosmic microwave background (CMB) [2]. Only in a narrow window around $70 \mathrm{GHz}$ does the CMB anisotropy signal dominate over synchrotron, dust and thermal bremsstrahlung emission from the Milky Way. For example, with a Galactic plane mask that preserves $77 \%$ of the sky the RMS CMB anisotropy begins to exceed the (falling) synchrotron foreground around $30 \mathrm{GHz}$ and drops below the (rising) foreground due to dust at about $160 \mathrm{GHz}$, while it dominates by a factor of $\sim 8$ between 70 and $80 \mathrm{GHz}$ - see Fig. 10 of [3]. In particular, detection of the elusive $B$-mode polarisation, a diagnostic of gravitational waves expected to have been generated during primordial inflation, requires detailed understanding and reliable subtraction of the Galactic 'foreground' [4-6]. This is also a limiting factor for the sensitivity of radio searches for dark matter annihilation signals as has been amply illustrated in the context of the 'WMAP haze' [7, 8].

As regards the GMF, it is customary to distinguish between an ordered and a 'random' component; the former is coherent on $\mathcal{O}(\mathrm{kpc})$ scales while the latter varies on scales of $\mathcal{O}(100)$ pc. Consequently, the specific synchrotron emissivity, i.e. the power emitted per unit volume and frequency, varies on two (disparate) scales. On small $(\lesssim 100 \mathrm{pc})$ scales the variation is almost entirely due to the turbulent nature of the interstellar magnetic field. It is believed that from the 'outer scale' ( $L \sim 100 \mathrm{pc}$ ) on which energy is injected into the interstellar medium (ISM) - most likely from old supernova remnant (SNR) shock waves - the turbulence cascades down to smaller scales. Both theoretical arguments $[9,10]$ and MHD simulations suggest a power law for the turbulent energy in magnetic fields:

$$
\mathrm{d} B(k)^{2} / \mathrm{d}^{3} k \propto k^{-\alpha}, \quad \alpha=11 / 3 .
$$

The spatial correlations of the magnetic field lead to angular correlations of the synchrotron flux $J, C(\psi) \equiv\left\langle J(\mathbf{n}) J\left(\mathbf{n}^{\prime}\right)\right\rangle$, where $\mathbf{n}$ and $\mathbf{n}^{\prime}$ are two directions on the sky and $\psi=$ $\arccos \left(\mathbf{n} \cdot \mathbf{n}^{\prime}\right)$ is the angle between them. A convenient representation for studying these correlations is the angular power spectrum (APS):

$$
\mathcal{C}_{l}=\int \mathrm{d}(\cos \psi) P_{l}(\cos \psi) C(\psi),
$$

i.e. the Legendre transform of the angular two-point correlation function $C(\psi)$ - structure on angular scales $\psi$ is encoded in multipoles $l \sim \pi / \psi$. The APS of a sky map $J(\theta, \phi)$ can also be calculated from its spherical harmonics coefficients $a_{l m}$ :

$$
\mathcal{C}_{l} \equiv \frac{1}{2 l+1} \sum_{m=-l}^{l}\left|a_{l m}\right|^{2} \quad \text { where } \quad a_{l m}=\int \mathrm{d} \Omega Y_{l m}^{*}(\theta, \phi) J(\theta, \phi) .
$$

This is equivalent to the definition in eq. (1.2) for a (statistically) isotropic sky; here, $Y_{l m}(\theta, \phi)$ denotes the spherical harmonics function.

For a power law spectrum of magnetic fluctuations (see eq.1.1), quasi-linear theory for wave-particle interactions predicts a rigidity-dependent diffusion coefficient which is also a power law with a related index, $D_{x x} \propto \mathcal{R}^{\delta}$ with $\delta=4-\alpha$ and a normalisation of $D_{x x} \sim$ $10^{28} \mathrm{~cm}^{2} \mathrm{~s}^{-1}$. The value of $\delta \sim 1 / 3$ corresponding to Kolmogorov turbulence is consistent 
with observations of nuclear secondary-to-primary ratios only in models with reacceleration, otherwise $\delta \sim 0.5$ fits better [11] (see however [12] who report that an even larger value $\delta \sim 0.8$ is possible). Therefore, for the energies relevant here, i.e. $E \lesssim 100 \mathrm{GeV}$, any structure in the distribution of $\mathrm{CR}$ electron sources is washed out by diffusion, such that the CR electron distribution is quite smooth on scales of $\lesssim 100 \mathrm{pc}$.

On large $(\gtrsim 1 \mathrm{kpc})$ scales, however, there are variations in both the CR electron distribution and the GMF. The variation of the electron density is determined by the spatial distribution of CR electron sources and the details of the propagation model, e.g. shape and extent of the propagation volume, diffusion coefficients and speed(s) of convective wind(s) if any. For the GMF, the ordered component is expected to follow the Galactic spiral arms (as is observed in other galaxies e.g. M82, M51, M81, NGC1068 and NGC6946), while the RMS value of its turbulent component is expected to decrease with distance, both from the Galactic centre and from the disk [13].

Attempts at modelling the diffuse synchrotron background have focussed on the distribution of emissivity on either very small or very large scales. It is known [14-17] that (assuming a statistically isotropic sky) the power law of the ISM turbulence is reflected in a broken power-law for the APS: below some critical multipole $l_{\mathrm{cr}}, \mathcal{C}_{l} \propto l^{-1}$, whereas for larger multipoles (smaller angular scales), $\mathcal{C}_{l} \propto l^{-\alpha}$. The critical multipole depends on the 'outer scale' of turbulence $L$ and the scale height $R$ of the turbulent medium: $l_{\mathrm{cr}} \sim 2 \pi R / L$. However, comparison with observations is hampered by the fact that the observed synchrotron sky is not statistically isotropic, due to large-scale variations of emissivity. Studies of MHD turbulence in the APS have therefore constrained themselves to patches of the sky, e.g. by cutting out bands around the Galactic plane. Clearly, this cannot describe the APS down to small $l$; in fact, attempts to fit the APS by a broken power law are able to reproduce the observed behaviour for $l \lesssim 20, l(l+1) \mathcal{C}_{l} \sim$ constant, only by assuming a small scale height, $R \lesssim 1 \mathrm{kpc}$ [18], which is quite unrealistic [19-21].

On large scales, two approaches have been adopted in the literature: Motivated by the need for a realistic model of the polarised emission, one set of studies [22-25] employ sophisticated models (of the ordered component) of the GMF constrained by detailed analyses of rotation measures (RM) and starlight polarisation, but make rather simplistic assumptions for the spatial distribution of relativistic electrons. The other set of studies [20, 26-28] start with a detailed model for CR propagation, reproducing locally measured spectra of protons, nuclei and electrons, and fix the (random component) of the GMF by fitting to the strength of the synchrotron radiation. Most of the emission on large scales would appear to be produced by the random component - in fact the magnitude of the ordered component as obtained from RM data is too weak to explain the radio flux, as has been recognised for some years [29]. However, the random component has not been modelled as a true random field but only in terms of its RMS value. While this modelling of the large-scale component can reproduce the global behaviour, there is mismatch on intermediate scales of tens of degrees. To downplay this, angular profiles of the radio background are usually averaged over large parts of the sky before comparison with data. The remaining discrepancies on smaller scales are usually attributed to "local structures" but there have been few attempts to model these.

It is thus necessary to model the emission consistently on both large and small angular scales. There must also be additional contributions at intermediate angular scales from the $\sim 1000$ old supernova remnants (SNRs) in the Galaxy which have expanded to large sizes of $\mathcal{O}(100)$ pc in the hot tenuous ISM before cooling and turning radiative [30]. Although these old SNRs may not actively accelerate CRs, they do compress the GMF in their 'supershells' 


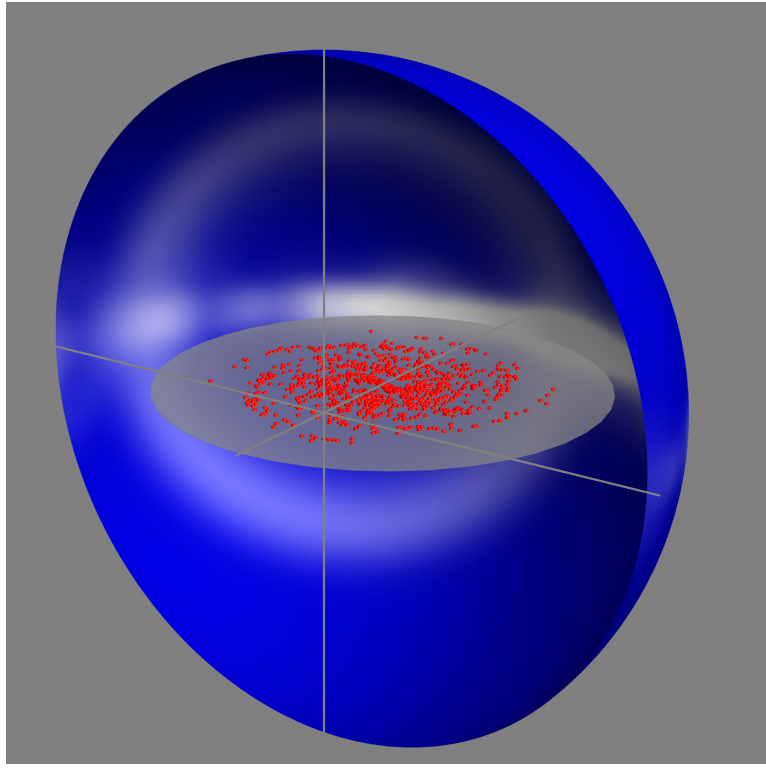

Figure 1. Sketch of the Galactic disk with shells of old supernova remnants and their skymap, i.e. their projection onto the celestial (hemi)sphere, in order to indicate the origin of 'loop' features in the radio sky. The (helio-centric) Galactic coordinate system is shown by the grey lines.

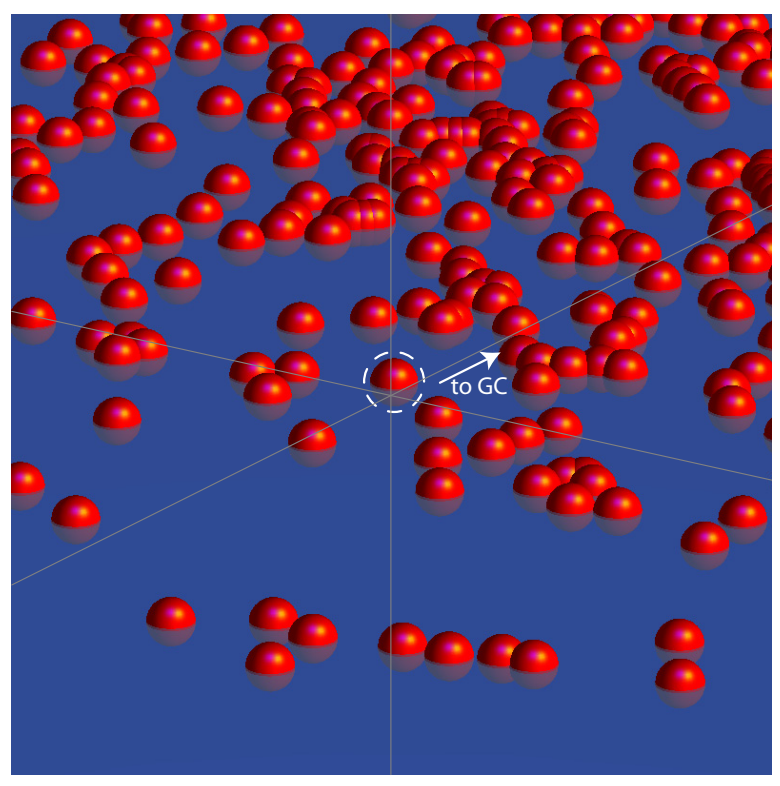

Figure 2. Zoom into the inner few kiloparsecs of the Galactic disk around the position of the Sun. The nearest old SNR which is responsible for the largest 'loop' feature on the radio sky is circled.

by large factors, and with it the tied CR electrons, which would moreover be then betatron accelerated to higher energies [31]. As the synchrotron emissivity scales as the square of the magnetic field strength and of the correlated increase in CR electron energy [32], the radio emission from such old SNR shells can be significantly amplified. It has been argued that if most of the Galactic 'diffuse' background arises from such (unresolved) old SNRs then the absolute emissivity can be explained with an average magnetic field strength of $\sim 2 \mu \mathrm{G}$, consistent with the RM measurements [33]. These large old SNRs may themselves generate the observed fluctuating component of the GMF. Given that they pervade the whole Galaxy and can be found at distances between hundreds of pc to tens of kpc, we expect them to contribute at angular scales between a few degrees up to the full sky (see Figs. 1 and 2).

In this paper, we attempt to model the radio sky on a wide range of scales, taking into account the large-scale contribution of CR electrons and of the GMF, the variation of the GMF due to the turbulence cascade in the ISM and the effect of the presence of large radiative shells of old SNRs. The observable we model is not the all-sky radio map itself but its APS, i.e. the distribution of $\mathcal{C}_{l}$ with $l$. Modelling the APS instead of sky maps has several advantages; firstly, the cascading of energy from the outer scale $L$ to the dissipation scale as well as the spatial distribution of old SNRs is a stochastic process of which the observed Galaxy represents only one particular realisation. What can be predicted are the ensemble averages which may to a certain degree differ from the observed Galaxy. The APS is a representation of this stochastic process that reduces the variation between different realisations by efficiently averaging over many lines of sight. Secondly, the APS presents a representation of the information sorted by angular size and also encodes the symmetry properties of the sky map. Finally, for CMB studies (and also a particular class of indirect 
searches for DM annihilation signals), the observable is also the APS and it is therefore favourable to perform the foreground subtraction not on sky maps but directly on the APS.

The remainder of this paper is organised as follows. In $\S 2$ we discuss the APS of the 'Haslam' $408 \mathrm{MHz}$ all-sky survey [34]. In $\S 3$ we present our model for the synchrotron background, discussing in detail each component (large-scale smooth emission, small-scale turbulence, intermediate scale old SNR shells) in turn. We demonstrate a good match of our model to the observed APS. In subsequent papers we will present the match to the observed frequency spectrum for different regions of the sky, as well as to the RM sky maps.

\section{The observed angular power spectrum}

We begin by computing the APS of the $408 \mathrm{MHz}$ all-sky survey [34]. Throughout this study, we used a verison of the map that has been corrected for scanning artefacts and with strong point sources removed. ${ }^{2}$ It is possible that unresolved point sources still contribute (in particular at high multipoles), however we will argue later that this is most likely negligible. Furthermore, as this is the only full-sky point-source subtracted map that is publicly available, we restrict ourselves, for the time being, to this one frequency. Important additional information is encoded in the frequency-dependence of the sky maps (or equivalently the APS), because of the different (and spatially varying) frequency dependence of the various physical components contributing to the diffuse emission, see $\S 3$. This is particularly true for synchrotron emission by relativistic electrons, given that the spatial distribution of the radiating CR electrons varies with energy due to their energy-dependent diffusion and energy loss rates. Extrapolating the APS (or sky maps, for that matter) between widely separated frequencies is therefore quite non-trivial [7]. However, if successful in developing a convincing physical model for the APS, we can be of course more confident in predicting the APS at other frequencies. It would therefore be desirable to test and (if necessary) calibrate our model by comparing to the APS at different frequencies. Here we adopt the complementary approach of fixing all the parameters using other observations, which allows for testing and dissecting the underlying (model) assumptions.

In Fig. 3, we show the APS of the $408 \mathrm{MHz}$ all-sky survey calculated using anaf ast from the HEALPix-suite [35]. For cosmological studies, it is customary to plot the $\mathcal{C}_{l}$ multiplied by $l(l+1) /(2 \pi)$ in order to facilitate comparison with a Harrison-Zeldovich primordial spectrum for which $\mathcal{C}_{l} \propto 1 / l(l+1)$. Doing so is still helpful in the present context since multiplying $\mathcal{C}_{l}$ by $l(l+1) \sim l(2 l+1)$ directly displays the power contained in logarithmic intervals of $l$.

It is seen that most of the power in the $408 \mathrm{MHz}$ map is present at multipoles $l \lesssim 10$, corresponding to angular scales $\gtrsim 20^{\circ}$. The most prominent feature in the APS, however, is the disparity between even and odd modes - a consequence of the (approximate) symmetry properties of the synchrotron map, in particular the symmetry between the northern and southern (Galactic) sky and the invariance with respect to shifts in longitude: If a sky map $J(\theta, \phi)$ is perfectly symmetric with respect to the Galactic plane, $J(\theta, \phi) \equiv J(\pi-\theta, \phi)$, then since the spherical harmonics satisfy $Y_{l m}(\pi-\theta, \phi)=(-1)^{l+m} Y_{l m}(\theta, \phi)$, it follows that $a_{l m}=(-1)^{l+m} a_{l m}$. If, in addition, we assume that the synchrotron flux is independent of longitude $\phi$, then all $a_{l m}$ vanish except when $m=0$. This results in the $a_{l m}$ for even $l$ (and $m=0$ ) being the only non-vanishing components, and therefore also the $\mathcal{C}_{l}$ s with even $l$. However, as the above assumptions are satisfied only approximately (in particular, invariance with respect to shifts in longitude), the symmetry is partially broken and the $\mathcal{C}_{l} \mathrm{~s}$

\footnotetext{
${ }^{2}$ http://lambda.gsfc.nasa.gov/product/foreground/fg_haslam_get.cfm
} 


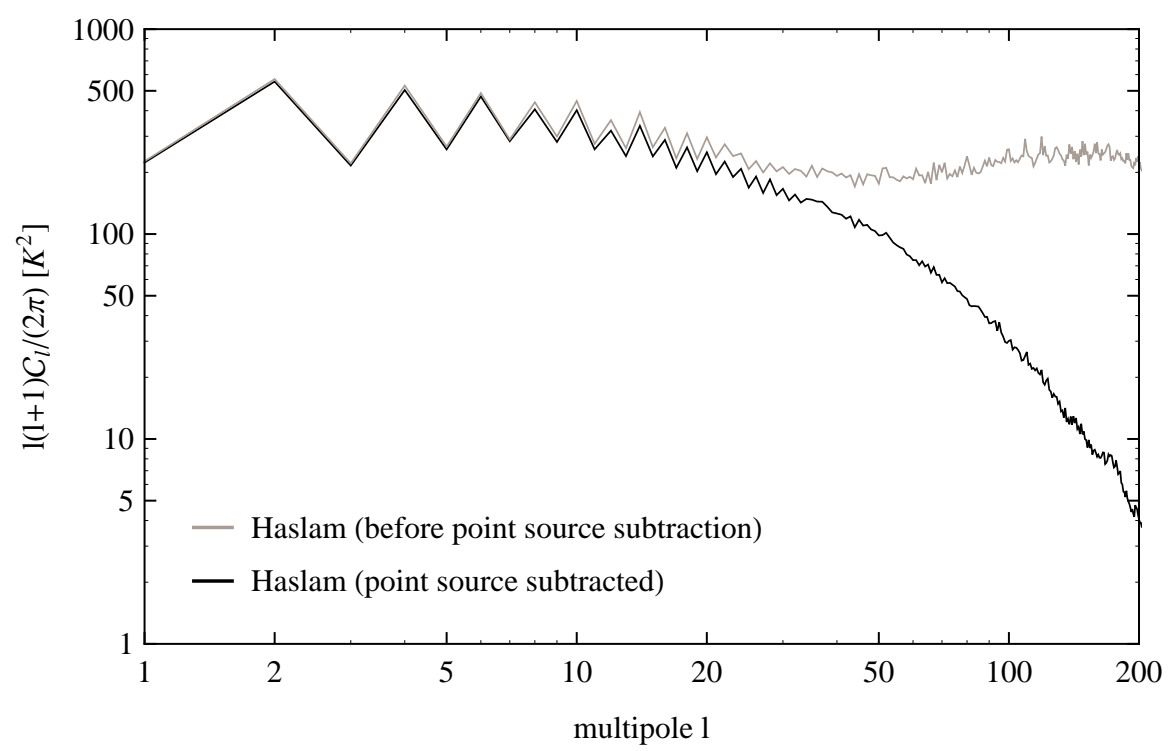

Figure 3. The APS of the $408 \mathrm{MHz}$ all-sky survey, both before and after correction for scanning artefacts and subtraction of point sources.

with odd $l$ become populated. Thus the ratio of odd to even $\mathcal{C}_{l}$ s is a measure of how much the Galactic plane symmetry is broken, and by how much the flux varies with longitude.

It is also apparent from Fig. 3 that this odd-even disparity does not extend to arbitrarily high multipoles. In fact, between $l=30$ and 40, the saw-tooth structure largely disappears, pointing at a different component dominating on smaller scales. Moreover the APS at large $(l \gtrsim 50)$ multipoles becomes rather smooth; this might seem surprising given how apparently stochastic structure is present in the sky maps on small scales. However, as mentioned already, the APS is formed at large $l$ by averaging over many different $m$-modes and correspondingly many different lines-of-sight (LoS), which leads to an efficient averaging of the stochastic information (i.e. the distribution of turbulent eddies or shells of old SNRs) and carves out the physically meaningful information that can actually be predicted, viz. their statistical expectation values.

\section{Modelling of the angular power spectrum}

\subsection{Definitions}

The power emitted at frequency $\nu$ per unit solid angle and frequency by an electron of energy $E$ in a magnetic field $\mathbf{B}$ is [36]:

$$
\varepsilon(\nu, E, \mathbf{B})=\frac{\sqrt{3} e^{3} B_{\perp}}{8 \pi^{2} \varepsilon_{0} c m_{e}}\left(\frac{\nu}{\nu_{c}}\right) \int_{\nu / \nu_{c}}^{\infty} \mathrm{d} x K_{5 / 3}(x) \quad \text { with } \quad \nu_{c}=\frac{3}{2} \gamma^{2} \frac{e B_{\perp}}{2 \pi m_{e}},
$$

where $B_{\perp}$ is the component of $\mathbf{B}$ perpendicular to the LoS, $e, m_{e}$ and $\gamma$ are, respectively, the electron charge, mass and relativistic gamma factor and $K_{5 / 3}$ is the Bessel function of degree $5 / 3$. Given a number $n_{e}(\mathbf{r}, E)$ of electrons per unit energy and volume at position $\mathbf{r}$ 


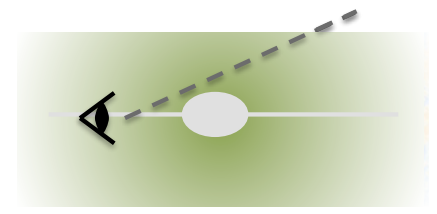

(a)

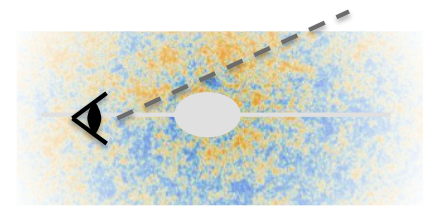

(b)

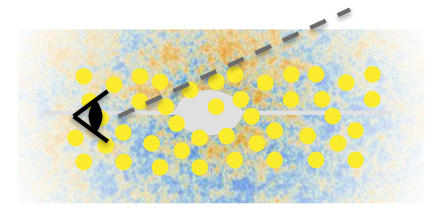

(c)

Figure 4. Different setups for calculations: (a) Smooth, large-scale emissivity with an off-center observer as considered in the GALPROP code (§ 3.2); (b) Turbulent halo dressed with the large-scale emissivity of the GALPROP code as in our MC calculation (§ 3.3); (c) As in panel (b) but with an additional contribution arising from a large number of old SNR shells (§ 3.6).

and with energy $E$, the total synchrotron power $\hat{\varepsilon}(\nu, \mathbf{r})$ is then

$$
\hat{\varepsilon}(\nu, \mathbf{r})=\int \mathrm{d} E n_{e}(E, \mathbf{r}) \varepsilon(\nu, E, \mathbf{B}(\mathbf{r})) .
$$

For a power law electron spectrum $n_{e}(E, \mathbf{r}) \propto E^{-\gamma}, \hat{\varepsilon}(\nu, \mathbf{r}) \propto \nu^{-(\gamma-1) / 2} B_{\perp}^{(\gamma+1) / 2}(\mathbf{r})$. For the locally measured $\gamma \simeq 3$, this gives: $\hat{\varepsilon}(\nu, \mathbf{r}) \propto \nu^{-1} B_{\perp}^{2}(\mathbf{r})$.

The flux $J(\nu ; \theta, \phi)$ observed in a particular direction $(\theta, \phi)$ on the sky is obtained by integrating the synchrotron emissivity $\hat{\varepsilon}(\nu, \mathbf{r})$ over the corresponding $\operatorname{LoS}, \mathbf{r}(s, \theta, \phi)$,

$$
J(\nu ; \theta, \phi)=\int_{0}^{\infty} \mathrm{d} s \hat{\varepsilon}(\nu, \mathbf{r}(s, \theta, \phi))=\int_{0}^{\infty} \mathrm{d} s \int \mathrm{d} E n_{e}(E, \mathbf{r}(s, \theta, \phi)) \varepsilon(\nu, E, \mathbf{B}(\mathbf{r}(s, \theta, \phi))) .
$$

In the following, we model the APS of the diffuse Galactic radio background, taking into account dynamics over a wide physical range: the emissivity of the ISM with variations on large (see Fig 4a) and small (see Fig 4b) scales as well as the shells of old supernova remnants (see Fig 4c). We also take into account free-free emission as well as the presence of unsubtracted point sources, although this is not the main thrust of this work.

\subsection{Large-scale variation of synchrotron emissivity}

We start by modelling the GMF on scales much larger than $L$ of $\mathcal{O}(100)$ pc, the assumed 'outer scale' of turbulence. For this we use the GALPROP code [37] which numerically solves the transport equation for Galactic cosmic rays (GCRs), taking into account the distribution of the sources (as determined from e.g. SNR or pulsar surveys), the spatial diffusion of CRs in the turbulent GMF, their convection by Galactic winds, various energy loss processes and 'reacceleration' due to diffusion in momentum space. We adopt the "pure diffusion model" z04LMPD [38], which is known to reproduce both the locally measured electron spectrum as well as the synchrotron spectrum averaged over large parts of the sky out of the Galactic plane. This model's parameter values can also reproduce locally measured GCR fluxes and secondary-to-primary ratios. While the height of the CR halo is important for the largescale distribution of the synchrotron emitting electrons and can therefore affect the lower 
multipoles, we have chosen to fix it to the best-fit-value $z_{\max }=4 \mathrm{kpc}[11]$ as we are not carrying out a full parameter study. Although the choice $\delta=0.5$ in z04LMPD is somewhat at odds with our assumed Kolmogorv spectrum for the ISM turbulence (which predicts $\delta=1 / 3$ ), we chose to retain it as a change of $\delta$ can easily be absorbed into the source spectral indices without changing the primary spectra too much. Note that as the assumed source distribution varies over distances $\gtrsim L$, so will the electron density. Rather than describe the turbulent GMF fully, we consider only its RMS value, averaged over ensembles of turbulent GMFs. For the normalisation and spatial dependence we adopt the model that is stated [19] to give a good fit to global angular profiles of the $408 \mathrm{MHz}$ all-sky survey, viz.

$$
B_{\text {rms }}(r, z)=B_{0} \mathrm{e}^{-r / \rho-|z| / \xi},
$$

with $B_{0}=7.5 \mu \mathrm{G}, \rho=30 \mathrm{kpc}$ and $\xi=4 \mathrm{kpc}$. Later we increase $\rho$ to $100 \mathrm{kpc}$ (i.e. essentially no variation with radius) to better reproduce the symmetry properties of the APS. We chose to ignore the ordered component altogether for the time being as its magnitude is much smaller than the RMS of the turbulent component. Note in this context, that due to the physically unavoidable correlations between magnetic field strength and electron density, the regular field as determined from synchrotron observations alone is likely overestimated [39], as was first pointed out many years ago $[32,33]$.

It has been recognised for some time [36] that the spectrum of the diffuse radio background softens between at few hundreds of $\mathrm{MHz}$ and a few $\mathrm{GHz}$, which probably reflects a break in the power-law describing the underlying electron spectrum. We follow $[19,21,40]$ in adopting a spectral break in the (source) electron spectrum at $\mathcal{R}_{1}=4 \mathrm{GV}$ (which has been also found to be necessary for fitting the spectra of protons and nuclei [11, 41]). This break has also been attributed to a change in the energy dependence of the diffusion coefficient $[42,43]$ but as the effect on the propagated spectrum is similar, we can remain agnostic as to its origin. The second break at $\mathcal{R}_{2}=50 \mathrm{GV}$ is probably not due to either cause but possibly the effect of an additional, harder contribution to the electron flux. (If the sources also accelerate positrons with a similar spectrum, this would explain the observed rise in the positron fraction [44].) The parameters adopted are shown in Table 1.

The synchrotron sky map is readily obtained from the GALPROP code in the HEALPix scheme and its APS is again calculated using the HEALPix suite.

\subsection{Small-scale variation of synchrotron emissivity}

Clearly the assumption that the GMF has structure only on scales larger than $L$ of $\mathcal{O}(100)$ pc is unrealistic. Firstly, the magnitude of the GMF as determined from Faraday rotation (which depends on the large-scale component, i.e. the GMF averaged over $\operatorname{LoS} \gtrsim L$ ) is too small [29] to reproduce the overall synchrotron flux (which depends on the mean square average value along the LoS). Hence, the variation on scales smaller than $L$ cannot be neglected. Secondly, a turbulent GMF field is also inferred from the fact that the observed anisotropy in the arrival directions of GCRs is very small: around $10^{-3}$ up to $100 \mathrm{TeV}$ and around $10^{-2}$ at $10 \mathrm{PeV}$ [46]. It is therefore believed that the dominant process of CR transport in the Galaxy is diffusion due to resonant wave-particle interactions - this requires a turbulent magnetic field on the scale of the particle gyroradius, e.g. $\sim 10 \mathrm{pc}$ for $E=10 \mathrm{PeV}$ in a $\mu \mathrm{G}$ field.

Under some simplifying assumptions the APS of the synchrotron emission from a turbulent magnetic field can be computed analytically [14]. Let the magnetic field be a Gaussian random field with an ensemble average that is only a function of the distance $s$ from the observer: $\left\langle\mathbf{B}^{2}\right\rangle \propto w(s)$ - we call this the statistically isotropic setup (see also Fig. 12). Let 


\begin{tabular}{|l|l|l|}
\hline Description & Parameter & Value \\
\hline \hline Spatial diffusion coefficient at 4 GV & $D_{0}$ & $3.4 \times 10^{28} \mathrm{~cm}^{2} \mathrm{~s}^{-1}$ \\
Power lax index of the spatial diffusion coefficient & $\delta$ & 0.5 \\
Half-height of CR propagation volume & $z_{\max }$ & $4 \mathrm{kpc}$ \\
\hline \hline \multirow{2}{*}{ Source distribution } & \multicolumn{2}{|l|}{ pulsar-like [45], but held } \\
& \multicolumn{2}{|c|}{ constant for $r>10 \mathrm{kpc}$} \\
\hline \multirow{2}{*}{ Source spectral indices for $\mathcal{R} \leq \mathcal{R}_{1}$} & $\Gamma_{1}$ & 1.6 \\
& $\Gamma_{2}$ & 2.5 \\
Break rigidities $\quad$ for $\mathcal{R}_{2}<\mathcal{R}$ & $\Gamma_{3}$ & 2.2 \\
& $\mathcal{R}_{1}$ & $4 \mathrm{GV}$ \\
\hline \hline Magnetic field strength at the Galactic centre & $\mathcal{R}_{2}$ & $50 \mathrm{GV}$ \\
Scale height in radial direction & $B_{0}$ & $7.5 \mu \mathrm{G}$ \\
Scale height in direction perpendicular to Galactic plane & $\rho$ & $30 \mathrm{kpc}, 100 \mathrm{kpc}$ \\
\hline
\end{tabular}

Table 1. Parameters of the propagation and magnetic field model [19, 38] adopted for the computation of the large-scale component of the synchrotron emission.

us approximate the electron spectrum as a power law $n(E) \propto E^{-3}$ which is close to the locally observed electron spectrum. The emissivity is then proportional to the square of the magnetic field perpendicular to the LoS which on average - assuming statistical isotropy - is $2 / 3$ of the square of the total magnetic field. It turns out that the $3 \mathrm{D}$ power spectrum of the random field (i.e. the Fourier transform of the two-point correlation function) is then reflected in the APS of the resulting synchrotron sky map [14]. In fact, if we assume a Kolmogorov power law with a cut-off at small wavenumber $k_{0}$ (corresponding to an outer scale of turbulence: $L \sim 1 / k_{0}$ ),

$$
\frac{\mathrm{d} B^{2}}{\mathrm{~d}^{3} k}=\mathcal{F}_{0}^{2} k^{-11 / 3} \mathrm{e}^{-k_{0}^{2} / k^{2}}
$$

If we further adopt $w(s)=\mathrm{e}^{-s^{2} / R^{2}}$, then for $l \gg 1$, the APS is a broken power law in $l$ :

$$
\mathcal{C}_{l}=2.96 \times 10^{3} \frac{\mathcal{F}_{0}^{4} R^{8 / 3}}{k_{0}^{2 / 3}} \frac{1+3.21 \mathrm{e}^{-1.43 l /\left(k_{0} R\right)}}{l^{11 / 3}\left(1+5.0\left(\frac{k_{0} R}{l}\right)^{2}\right)^{4 / 3}},
$$

with power law indices $-1(-11 / 3)$ for $l \lesssim l_{\text {cr }}\left(l \gtrsim l_{\text {cr }}\right)$. The break occurs around a critical multipole that depends on the path length $R$ and the outer scale of turbulence $L$ as $l_{\text {cr }} \approx$ $(2 \pi R / L)[14-16]$.

It has been argued that the observed APS follows a power law at large $l$ with spectral index $-11 / 3$ due to this small-scale variation of synchrotron emissivity $[14-16,18]$. However, for this to hold the normalisation of the observed high- $l$ component should also be reproduced. In fact, in the statistically isotropic setup, there is a close relation between the monopole $\mathcal{C}_{0}$ and the power of the higher $(l>0)$ multipoles. It is shown in Appendix A however that the power in the monopole is always many orders of magnitude larger than the power contributed by the rest of the APS. Therefore, fixing the normalisation $\mathcal{F}_{0}^{2}$ in eq. (3.5) to reproduce the APS at large $l$ from eq. (3.6) will imply too large a monopole. We conclude that the magnetic turbulence can contribute very little to the APS at large $l$. 
Of course the real question is how the picture changes when we consider a more realistic setup, viz. an off-center observer in a medium with small-scale turbulence, that is dressed with the overall large-scale emissivity of the GALPROP run from $\S 3.2$ (cf. Fig. 4b). One would then expect the APS to be more-or-less the sum of the large-scale and the small-scale contributions as these are on disjoint scales. For the small-scale component, one would need to average the APS over a distribution of scale-lengths $R$ since the scale length in the offcenter setup depends on the direction [15]. Moreover since the large-scale emissivity is no longer isotropic, the monopole computed in (A.2) gets spread out into the lowest multipoles and we would therefore expect the large-scale power in these lowest multipoles to dominate over the small-scale power at the higher multipoles.

We have computed the sky map and its APS numerically by a Monte Carlo calculation. To that end we have prepared a turbulent magnetic field by superposing hundreds of plane waves evenly spaced logarithmically in $k$ with amplitudes chosen so as to reproduce a power law power spectrum $\propto k^{-11 / 3}$ with unit variance for $k>2 \pi / L$ [47]. In particular, we adopt a coherence length $L=420 \mathrm{pc}$. This statistically isotropic Gaussian random field $\mathbf{b}(\mathbf{r})$ is then considered as the deviation of the turbulent magnetic field $\mathbf{B}(\mathbf{r})$ from its ensemble average $B_{\text {rms }}(r, z)$ (eq. 3.4), i.e. $\mathbf{B}(\mathbf{r})=B_{\text {rms }}(r, z) \mathbf{b}(\mathbf{r})$. Given the (smooth) emissivity (cf. $\S 3.3$ ) calculated by GALPROP, $\hat{\varepsilon}\left(\nu ; B_{\mathrm{rms}}(r, z)\right)$, we can now compute the emissivity from the fully turbulent field by using the scaling of the emissivity and critical frequency with the magnetic field $B$ (cf. eq. 3.1),

$$
\varepsilon(\nu ; \beta B)=\beta \varepsilon(\nu / \beta ; B)
$$

where $\beta=\left|\mathbf{b}_{\perp}(\mathbf{r})\right|$. We generate a sky map by integrating this emissivity along LoS's over the whole sky and calculate its APS as above. In Fig. 5 we show that the dependence on the value of $L$ is rather mild.

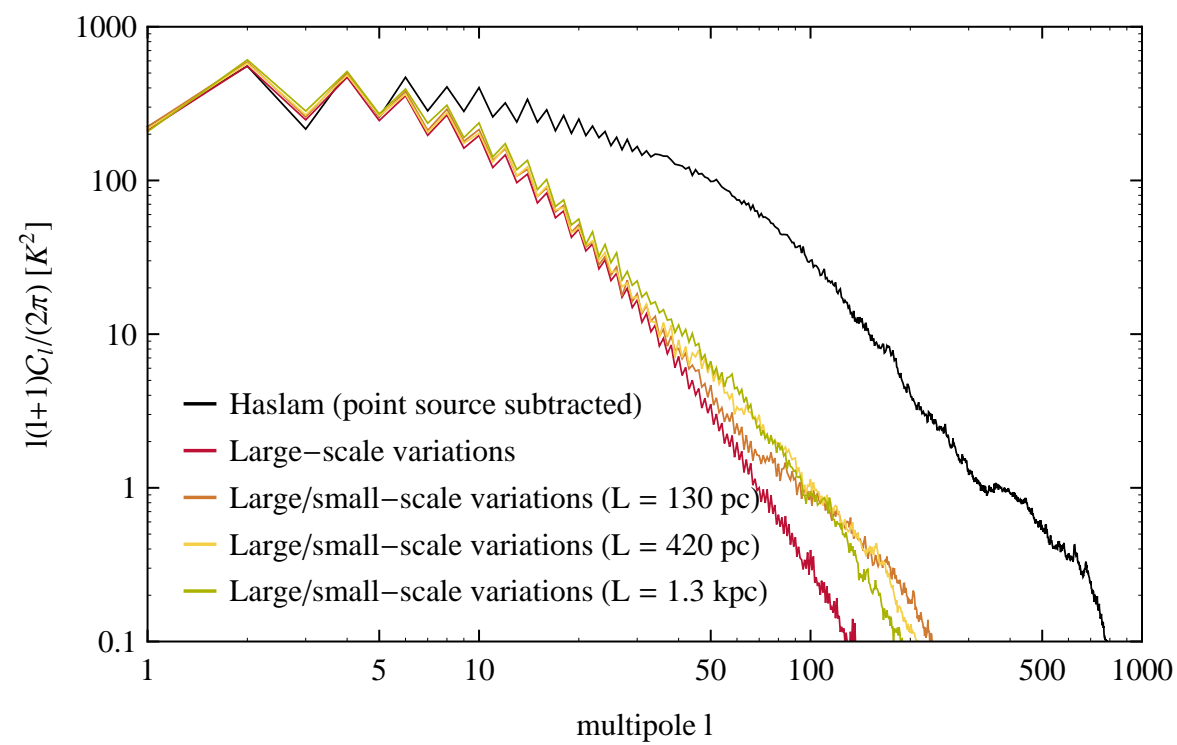

Figure 5. The APS of radio emission due to small-scale turbulence in the Galactic magnetic field for different choices of the 'outer scale' $L$. 


\subsection{Free-free emission}

Free-free emission is the bremsstrahlung of thermal electrons in the ISM. For an electron temperature $T_{e}$ (in units of degrees Kelvin), the optical depth is given by [48]

$$
\tau_{c}=3.014 \times 10^{-2} T_{e}^{-3 / 2} \nu^{-2}\left(\ln \left[4.955 \times 10^{-2} \nu^{-1}\right]+1.5 \ln T_{e}\right) \int_{\mathrm{LoS}} \mathrm{d} \mathbf{r} n_{e} n_{i}
$$

and the brightness temperature is $\propto \tau_{c} T_{e}$. A reasonable approximation is

$$
\tau_{c} \propto T_{e}^{-1.35} \nu^{-2.1} \int_{\mathrm{LoS}} \mathrm{d} \mathbf{r} n_{e} n_{i}
$$

and the brightness temperature then factorises into a frequency part $\left(\propto \nu^{-2.1}\right)$ and a spatial/angular part.

Consequently, unlike synchrotron emission the spatial distribution of thermal bremsstrahlung emissivity is not expected to change much with frequency (unless the ISM electron temperature varies significantly between locations). We can therefore attempt to trace the free-free emission with a template. Here, we employ the estimate for the free-free emission at $23 \mathrm{GHz}$ provided by the WMAP team using a maximum entropy method (MEM) [49] and their adopted frequency spectral index of -2.15 .

We wish to emphasise however that the MEM free-free template is not an unbiased tracer of thermal bremsstrahlung. First, it was derived by separating the observed $23 \mathrm{GHz}$ sky map into synchrotron, free-free and thermal dust emission and therefore any error in the synchrotron extrapolation translates into errors on the MEM free-free template. Moreover, spinning dust emission was ignored all together such that the MEM templates potentially compensate for this. The template therefore likely overestimates the real free-free emission. Alternatives to the MEM template, e.g. the WMAP MCMC model, have been shown however to be even less reliable [27] and over- (under-)estimate free-free emission when excluding (including) a spinning dust component. The MEM free-free template on the other hand has a rough correlation with maps of radio recombination lines so we adopt it here but with the above reservations.

\subsection{Unsubtracted point sources}

It is possible that the point source subtractions performed on the $408 \mathrm{MHz}$ all-sky survey have not been able to subtract all the sources present in the sky map. Relatively faint sources, and even relatively strong sources in the Galactic disk, might have been missed. Here, we model their APS by shot noise, as is expected for an unclustered population of point-like objects (see [50]), and fix the normalisation such that the observed APS is matched at the highest $l$ accessible, i.e. $l \sim 200$. We find that $\mathcal{C}_{l} \sim 2 \times 10^{-2} \mathrm{~K}^{2}$ allows for a good fit at large $l$. By comparing the APS of the unsubtracted with that of the (partly) subtracted $408 \mathrm{MHz}$ all-sky survey, we have confirmed that this indeed describe the APS of the point sources rather well.

\subsection{Shells of old SNRs}

We will see in the next section that when the APS is modelled by adding contributions from both large- and small-scale variations of synchrotron emissivity, as well as free-free emission and unsubtracted point sources, there is still a large deficit in power with respect to the observed APS on intermediate scales $(10 \lesssim l \lesssim 100)$. This cannot be explained either by an increase in free-free emission, nor the presence of unsubtracted point sources. However, a 
class of extended sources that would contribute to the APS on such scales is visible already in sky maps from radio to microwaves (and even in gamma-rays): the so-called 'radio loops' [51]. These huge ring-like structures, that span up to $60^{\circ}$ in angular radius, are believed to be the large - up to $\sim 200 \mathrm{pc}$ in radius - shells of old SNRs (see $[52,53]$ for detailed studies of Loop I, also called the North Polar Spur).

Towards the end of its lifetime, a SNR is in its radiative phase with a low expansion velocity of $\mathcal{O}(100) \mathrm{km} \mathrm{s}^{-1}$ such that particle acceleration is no longer expected to take place (see [54]). However, as the expansion is now in the pressure-driven 'snow plough' phase [55], the shock compression factor $\eta$ can get very large leading to a strong enhancement of the ISM magnetic field upon crossing the shock. In the ISM there will be a probability distribution of $\eta$, as was computed [33] for the McKee-Ostriker model, however for simplicity we adopt a median value $\eta=6$ which is conservative. This has two important consequences [31,33]: First, by betatron acceleration the energy of electrons increases by a factor $\sim \sqrt{\eta}$ upon crossing the shock; as the electron spectrum is a steeply falling power law, this increases the emissivity for a fixed frequency. Second, due to the compression of the magnetic field by the same factor $\eta$, the emissivity at fixed frequency is now sourced by electrons which are a factor $\eta$ lower in energy - since these are more numerous, this further increasing the emissivity. Hence, a compressed shell easily outshines the emissivity of the surrounding ISM.

Given at set of shells at positions $\left\{\mathbf{r}_{i}\right\}$, the total synchrotron flux $J_{\text {shells }}(\nu ; \ell, b)$ is the sum over all individual shells $i$, and so are the $a_{l m}$ :

$$
J_{\text {shells }}(\nu, \ell, b)=\sum_{i} J_{\text {shell } i}(\nu, \ell, b) \quad \Rightarrow \quad a_{l m}^{\text {shells }}=\sum_{i} a_{l m}^{\text {shell } i} .
$$

We approximate the compressed medium of an old SNR as a spherical shell of inner (outer) radius $R_{1}\left(R_{2}\right)$ with a constant compression factor $\eta$. Then the flux from a single shell $i$ centred at at $\mathbf{r}_{i}$ factorises into a frequency-dependent specific emissivity $\varepsilon_{i}(\nu)$ and an angular LoS integral $g_{i}(l, b)$ :

$$
J_{\text {shell } i}(\nu, \ell, b)=\underbrace{\int \mathrm{d} E n_{\text {acc'd }}\left(E ; \mathbf{r}_{i}\right) \varepsilon\left(\nu, \eta \mathbf{B}\left(\mathbf{r}_{i}\right), E\right)}_{\varepsilon_{i}(\nu)} \underbrace{\int \mathrm{d} s \rho\left(\left|\mathbf{r}(s, \theta, \phi)-\mathbf{r}_{i}\right|\right)}_{g_{i}(l, b)},
$$

where $\rho(r)=1$ for $R_{1} \leq r \leq R_{2}$ and 0 otherwise. Here, we have approximated both the betatron-accelerated electron spectrum $n_{E ; \text { acc'd }}(\mathbf{r})$ and magnetic field $\mathbf{B}(\mathbf{r})$ in the shell by their values at its centre $r_{i}$, i.e. $n_{\text {acc'd }}\left(E ; \mathbf{r}_{i}\right)$ and $\mathbf{B}\left(\mathbf{r}_{i}\right)$.

\subsubsection{Specific emissivity of a shell}

Consider an electron with pitch angle $\theta_{0}$, i.e. momentum $p_{\perp}=p \sin \theta_{0}\left(p_{\|}=p \cos \theta_{0}\right)$ perpendicular (parallel) to the magnetic field. Due to efficient compression of the GMF, the magnetic field in the shell will be mostly aligned with the shock, that is tangential to the shell. If the shock width is larger than the gyroradius of the $(\mathrm{GeV})$ electron, the time-scale over which the magnetic field $B$ changes is larger than a gyration period, and therefore the first adiabatic invariant $p_{\perp}^{2} / B$ is conserved. Upon crossing the shock with compression factor $\eta$, the electron therefore gains a factor $\sqrt{\eta}$ in $p_{\perp}$, while $p_{\|}$remains constant. An initially isotropic distribution $n(p)$ therefore becomes anisotropic:

$$
n(p) \rightarrow n_{\text {acc'd }}(p, \theta)=n\left(\frac{p}{\sqrt{1+(\eta-1) \sin ^{2} \theta_{0}}}\right) .
$$


where the new pitch angle $\theta$ is a function of the initial pitch angle $\theta_{0}$ (see [31] for details). Following [33] we assume that this distribution gets quickly isotropised by pitch-angle scattering on Alfvén waves behind the shock. The distribution then needs to be averaged over the new pitch angle $\theta$, however, an order of magnitude approximation is given by:

$$
n_{\text {acc'd }}(p, \theta) \approx \frac{2}{\sqrt{\eta}} n\left(p \sqrt{\frac{3}{2 \eta}}\right),
$$

assuming that only the two perpendicular components of the momentum change. We note that the diffusive escape of electrons from the shells can be neglected as the magnetic field at the edge is tangential and perpendicular diffusion is much suppressed.

The emissivity $\hat{\varepsilon}_{\text {acc'd }}$ at frequency $\nu$ of the compressed shell can now be simply expressed in terms of the nominal emissivity $\hat{\varepsilon}$ of the (unaccelerated) electrons in the (uncompressed) magnetic field, but at a different frequency:

$$
\begin{aligned}
\hat{\varepsilon}_{\text {acc'd }}(\nu, \eta B) & =\int \mathrm{d} E \frac{2}{\sqrt{\eta}} n\left(E \sqrt{\frac{3}{2 \eta}}\right) \varepsilon(\nu, \eta B, E) \\
& =\sqrt{\frac{8}{3}} \int \mathrm{d} E^{\prime} n\left(E^{\prime}\right) \varepsilon\left(\nu, \eta B, \sqrt{\frac{2}{3} \eta E^{\prime}}\right) \\
& =\sqrt{\frac{8}{3}} \eta \int \mathrm{d} E^{\prime} n\left(E^{\prime}\right) \varepsilon\left(\frac{3 \nu}{2 \eta^{2}}, B, E^{\prime}\right) \\
& =\sqrt{\frac{8}{3}} \eta \hat{\varepsilon}_{\mathrm{ISM}}\left(\frac{3 \nu}{2 \eta^{2}}, B\right),
\end{aligned}
$$

where we have used the scaling of the specific synchrotron emissivity (see eq. 3.1).

\subsubsection{Line-of-sight integral over a shell}

The projection of the shell onto the celestial sphere is a limb-brightened disk and for a given distance $d_{i}$, the flux depends on the angular distance $\theta^{\prime}=\arccos z^{\prime}$ from the projected centre of the sphere only (see Figs. 6 and 7):

$$
g_{i}\left(z^{\prime}\right)=2\left\{\begin{array}{llr}
\sqrt{R_{2}^{2}-d_{i}^{2}\left(1-z^{\prime 2}\right)}-\sqrt{R_{1}^{2}-d_{i}^{2}\left(1-z^{\prime 2}\right)} & \text { for } & \sqrt{d_{i}^{2}\left(1-z^{\prime 2}\right)} \leq R_{1}, \\
\sqrt{R_{2}^{2}-d_{i}^{2}\left(1-z^{\prime 2}\right)} & \text { for } R_{1}<\sqrt{d_{i}^{2}\left(1-z^{\prime 2}\right)} \leq R_{2}, \\
0 & \text { otherwise }
\end{array}\right.
$$

We start by considering the $a_{l m}^{\prime i}$ of an individual shell $i$ in a coordinate system $O^{\prime}$ with $z^{\prime}$-axis in the direction of the centre of the shell (see Fig. 6),

$$
\begin{aligned}
a_{l m}^{\prime i} & =\varepsilon_{i}(\nu) \int \mathrm{d} \Omega^{\prime} Y_{l m}^{*}\left(\theta^{\prime}, \phi^{\prime}\right) g\left(z^{\prime}\right) \\
& =\varepsilon_{i}(\nu) \int_{-1}^{1} \mathrm{~d} z^{\prime} \int_{0}^{2 \pi} \mathrm{d} \phi^{\prime} \sqrt{\frac{2 l+1}{4 \pi}} \sqrt{\frac{(l-m) !}{(l+m) !}} P_{l}^{m}\left(z^{\prime}\right) \mathrm{e}^{i m \phi^{\prime}} g\left(z^{\prime}\right) \\
& =\left\{\begin{array}{lll}
\varepsilon_{i}(\nu) \sqrt{\frac{2 l+1}{4 \pi}} \int_{-1}^{1} \mathrm{~d} z^{\prime} P_{l}\left(z^{\prime}\right) g\left(z^{\prime}\right) & \text { for } & m=0, \\
0 & \text { otherwise. }
\end{array}\right.
\end{aligned}
$$




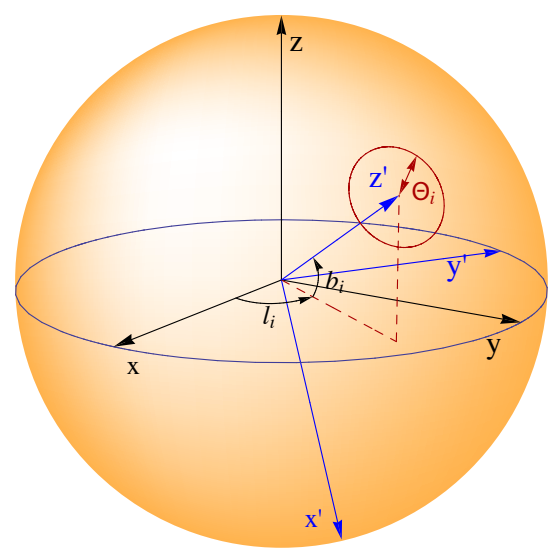

Figure 6. Coordinate systems used for the computation of the APS of the shells. The black, unprimed coordinate system $O$ is heliocentric with the Galactic centre at $(x, y, z)=(8.5,0,0) \mathrm{kpc}$, and the Galactic north pole in positive $z$-direction. The blue, primed coordinate system $O^{\prime}$ is centred on the direction of the centre of a shell and can be obtained by a rotation of $\ell_{i}$ around the $z$ axis and a rotation of $\pi / 2-b_{i}$ around the $y^{\prime}$-axis.

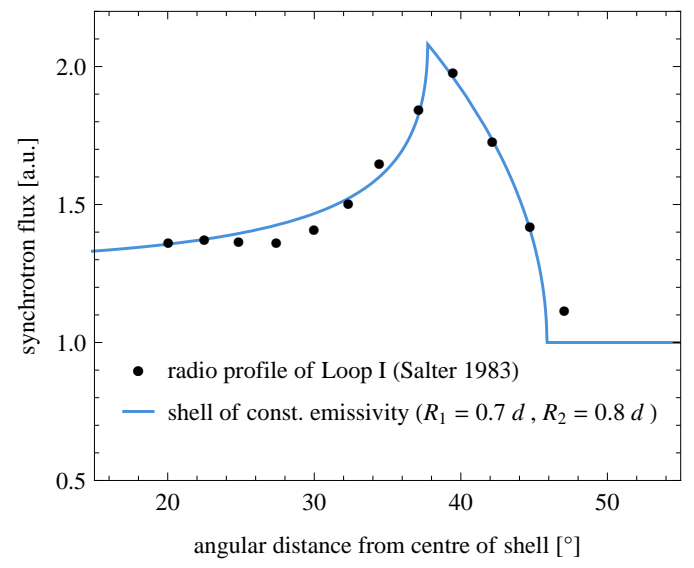

Figure 7. Angular profile of a shell of an old SNR. The data points show the (frequency averaged) angular radio profile as measured in Loop I [52]. The solid line is the profile of a shell of constant volume emissivity with inner radius $R_{1}=0.7 d$ and outer radius $R_{2}=0.8 d$.

The spherical harmonics coefficients $a_{l m}^{i}$ in Galactic coordinates $O$ can be computed using the Wigner D-matrices $D_{l}^{m^{\prime} m}\left(-\ell_{i}, \pi / 2-b_{i}, 0\right)$,

$$
\begin{aligned}
a_{l m}^{i} & =\varepsilon_{i}(\nu) \sum_{m^{\prime}=-l}^{l} D_{l}^{m^{\prime} m}\left(-\ell_{i}, \frac{\pi}{2}-b_{i}, 0\right) a_{l m^{\prime}}^{i} \\
& =\varepsilon_{i}(\nu) D_{l}^{0 m}\left(-\ell_{i}, \frac{\pi}{2}-b_{i}, 0\right) \sqrt{\frac{2 l+1}{4 \pi}} \int_{-1}^{1} \mathrm{~d} z^{\prime} P_{l}\left(z^{\prime}\right) g\left(z^{\prime}\right) \\
& =\varepsilon_{i}(\nu) Y_{l m}^{*}\left(\frac{\pi}{2}-b_{i},-\ell_{i}\right) \int_{-1}^{1} \mathrm{~d} z^{\prime} P_{l}\left(z^{\prime}\right) g\left(z^{\prime}\right) .
\end{aligned}
$$

The integral in the last line can be written:

$$
\int_{-1}^{1} \mathrm{~d} z^{\prime} P_{l}\left(z^{\prime}\right) g\left(z^{\prime}\right)=d J_{l}\left(\sqrt{1-\left(R_{2} / d\right)^{2}}\right)-d J_{l}\left(\sqrt{1-\left(R_{1} / d\right)^{2}}\right),
$$

where

$$
J_{l}(a)=\int_{a}^{1} \mathrm{~d} z^{\prime} P_{l}\left(z^{\prime}\right) \sqrt{z^{2}-a^{2}}
$$

can be solved analytically in terms of Gauss hypergeometric functions ${ }_{2} F_{1}$ (cf. App. B).

\subsubsection{The Galactic population of radio loops}

Only four of the radio loops are visible in the sky and can be modelled directly. We fix their radius to $200 \mathrm{pc}$ which is consistent both with calculations of the evolution of SNRs $[55,56]$ 
and estimates for Loop I $[52,53]$. We have adopted the positions and angular sizes as found originally [51] and computed the distances, see Table 2.

\begin{tabular}{|l|l|l|l||l|}
\hline Object & $l$ (centre) & $b$ (centre) & diameter & distance \\
\hline Loop I & $329^{\circ}$ & $17.5^{\circ}$ & $116^{\circ}$ & $240 \mathrm{pc}$ \\
Loop II & $100^{\circ}$ & $-32.5^{\circ}$ & $91^{\circ}$ & $280 \mathrm{pc}$ \\
Loop III & $124^{\circ}$ & $15.5^{\circ}$ & $65^{\circ}$ & $370 \mathrm{pc}$ \\
Loop IV & $315^{\circ}$ & $48.5^{\circ}$ & $39.5^{\circ}$ & $590 \mathrm{pc}$ \\
\hline
\end{tabular}

Table 2. Angular positions, diameters and resulting physical distances of the local radio loops for an assumed physical radius of $200 \mathrm{pc}$ [51].

However, the observable loops constitute most likely only a small fraction of the total number of old SNRs in the Galaxy. Adopting a SN rate of $1-3 \times 10^{-2} \mathrm{yr}^{-1}$ and estimating the duration of the radiative phase to be between $10^{4}-10^{5} \mathrm{yr}$, we can expect up to thousands of those old SNR shells to contribute to the synchrotron emission in radio and microwaves. Most of them will however not be directly observable in the sky since being further away than the 4 local loops they will blend into the diffuse emission from the Milky Way [33]. We therefore model them by Monte Carlo by randomly choosing 1000 positions distributed according to the pulsar-like source distribution adopted by GALPROP (see Sec. 3.2 and Table 1). As we will add the contribution from this random set to that from the known local loops, we have excised from the random set all those shells that are closer than $500 \mathrm{pc}$ in order not to double-count the local shells.

We fix the radius of all shells to $200 \mathrm{pc}$ and calculate their spherical harmonics expansion coefficients $a_{l m}^{i}$ from eqs. 3.17 and 3.24. Finally, we sum up the spherical harmonics expansion coefficients to $a_{l m}^{\text {shells }}$ and compute their angular power spectrum $\mathcal{C}_{l}^{\text {shells }}$.

\section{Results}

\subsection{APS from conventional model}

In Fig. 8 we show our modelling of the angular power spectrum resulting from both largescale and small-scale variations of synchrotron emissivity as well as free-free emission and unsubtracted point sources and compare it to the observed APS of the $408 \mathrm{MHz}$ all-sky survey. For all modelled components we have taken into account the exponential cut-off due to the beam window function.

We start the discussion by computing the APS from the variation of the synchrotron emissivity on large scales only (orange line). We have normalised this component by matching the dipole $(l=1)$ which required decreasing the intensity by $28 \%$. We note that most of the power is indeed contained on large scales $(l \leq 10)$ and the APS falls off quite rapidly for higher $l$, as is indeed expected given that all the ingredients (source distribution, transport parameters and magnetic fields) vary only on kpc scales. The APS computed with the parameters of $[19,38]$ does show the expected even-odd multipole structure but the difference between the power in even and odd multipoles is too small. This signals that the distribution in longitude is not extended enough; if it were more extended this would bring us closer to the symmetric case and would suppress power in the even multipoles. In Fig. 8 and henceforth we have therefore adopted $\rho=100 \mathrm{kpc}$.

Next we compare the contribution of the ISM synchrotron emissivity, with (red line) and without (orange line) the small-scale turbulence. We note that while the turbulent APS 

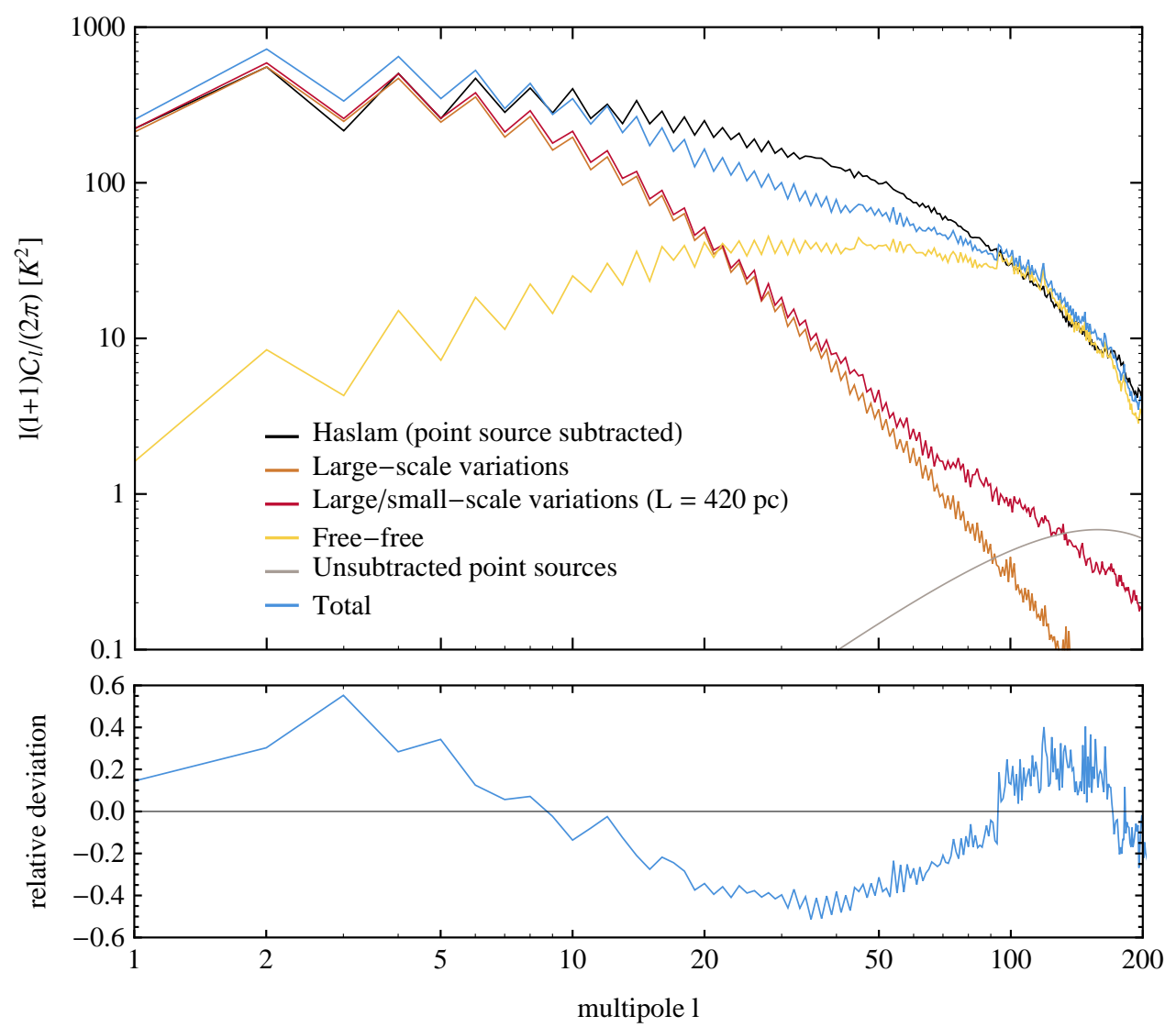

Figure 8. Top panel: The APS of the synchrotron sky computed by taking into account the large-scale and small-scale variations of emissivity (orange line), free-free emission (purple line) and unsubtracted sources (grey line). The sum of these components (blue line) is compared with the APS from the $408 \mathrm{MHz}$ all-sky survey (black line). Bottom panel: The relative residual of the modelled APS with respect to the observed one.

indeed shows a power law behaviour $\mathcal{C}_{l} \propto l$ for $l \gtrsim 50$, the additional power due to turbulence is relatively small. This corroborates our arguments from $\S 3.3$ about the relative power in the monopole and in higher multipoles and demonstrates that the large-scale behaviour of the measured APS cannot be due to ISM turbulence.

Now adding the contribution from free-free emission (yellow line) and the $\mathcal{C}_{l}$ due to unsubtracted sources (grey line, almost negligible below $l \sim 100$ ), we arrive at the total APS (blue line). Comparing this to the measured APS, it is evident that ISM synchrotron and free-free emission alone can give a reasonable fit only for the dipole and quadrupole and around $l \sim 100$. At all other $l$, and in particular at intermediate multipoles, the model underproduces the power observed in the $408 \mathrm{MHz}$ all-sky survey.

\subsection{APS adding SNR shells}

Figure 9 shows the modelled APS as before, but now including the contribution from the four local plus the $\sim 1000$ shells distributed in the Galaxy, for 10 different realisations of this 


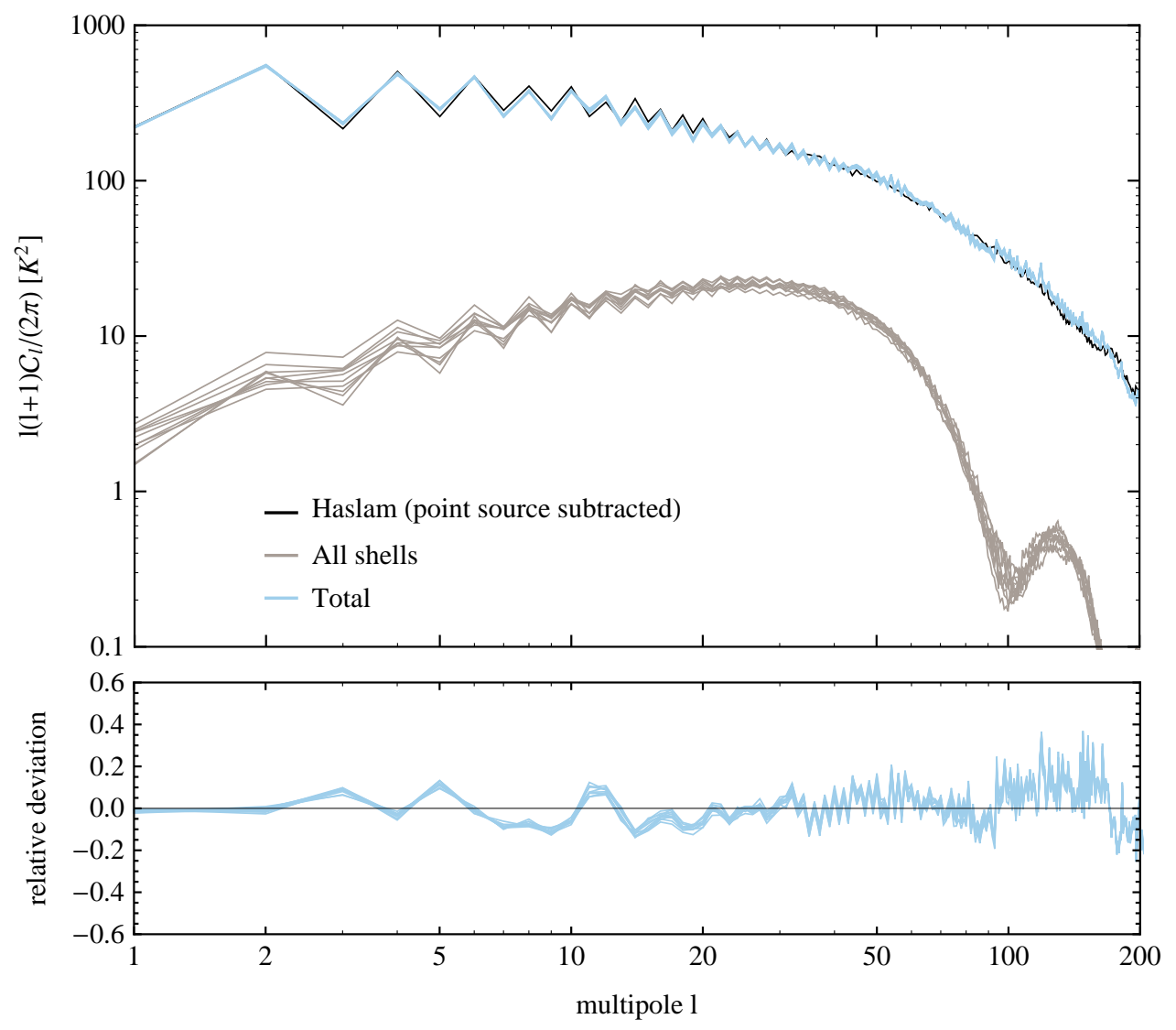

Figure 9. Top panel: The APS of the synchrotron sky computed by summing the large-scale and small-scale variations of emissivity, free-free emission, unsubtracted sources as well as the contribution from different configurations of shells of old SNRs (also shown separately). Bottom panel: The relative residual of the modelled APS with respect to the observed one.

ensemble. The APS for the shells alone is shown to illustrate that these contribute on large and intermediate scale, i.e. at $l \lesssim 50$ which is where we previously noted a deficit. (As the shells were added to the GALPROP model, we needed to further renormalise the contribution from the ISM emissivity to about $70 \%$.) The agreement with the measured APS is now much improved with residuals at most $20 \%$. The differences between the Monte Carlo realisations of the Galactic distribution of old SNRs is relatively small which illustrates our point that the APS is particularly appropriate for modelling of Galactic foregrounds as the influence of unknown parameters (e.g. the positions of and distances to the shells) is rather mild. Since the shells do not dominate the power at any $l$, the slightly larger residuals for $l \gtrsim 100$ must be attributed to the free-free MEM map, concerning which we have expressed some reservations already.

In Fig. 10 we show the best fit of our simple model to the observed APS - this has residuals no bigger than $10 \%$ up to $l \sim 200$, i.e. down to a degree. 

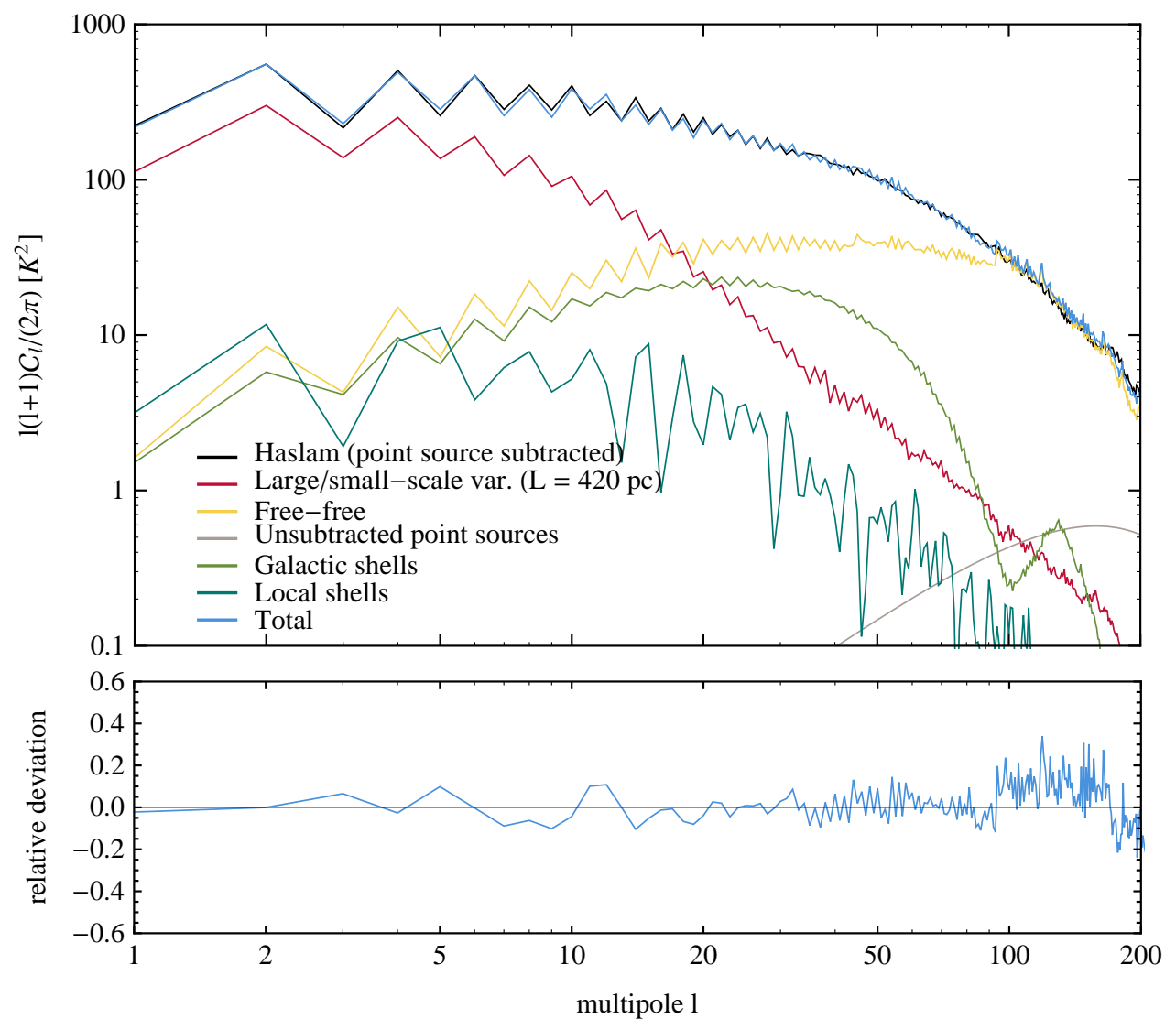

Figure 10. Top panel: The APS of the synchrotron sky obtained by summing the large-scale and small-scale variations of emissivity, free-free emission, unsubtracted sources, as well as the contribution from the best fit configuration of shells of old SNRs (also shown separately). Bottom panel: The relative residual of the modelled APS with respect to the observed one.

\section{Discussion}

The reader might wonder why we attempt to develop a physical model of the Galactic synchrotron emission in order to e.g. perform a reliable foreground subtraction for the CMB, since it is supposedly possible to measure CMB temperature anisotropies successfully even without a physical model of the foregrounds [57]. This is justified by saying that most foregrounds (in particular on small scales, i.e. at high $l$ ) are contained in the Galactic disk and can therefore be suppressed by simply masking this part of the sky. However apart from complicating the analysis (spherical harmonics on the cut sky are not orthonormal, thus introducing correlations between the $a_{l m}$ of different $l$ ), it is hard to estimate by how much the masked sky maps are still contaminated by high-latitude Galactic foregrounds. It is quite possible that the low multipole anomalies of the CMB are at least partly due to such residual contamination. Even in e.g. the WMAP 9-year data, the cosmological model fit is particularly poor at low multipoles and this is acknowledged "to be at least partially due to residual foregrounds" [58].

Second, our modelling of the unpolarised synchrotron emission is only a first step, with 
the next challenge being a sound understanding of the polarised component which will need to be subtracted off for measurement of the CMB $B$-mode polarisation due to gravitational waves from inflation. At $90 \mathrm{GHz}$ the expected signal for a realistic value of the tensor-toscalar ratio $r \sim 0.01$ reaches a maximum of $\sim 10-30 \%$ of the estimated foreground on degree scales, over $75 \%$ of the sky [4]. Hence to reliably extract this will require an understanding of the polarised foreground at the $10 \%$ level. Our fit to the unpolarised emission is already accurate to $10 \%$ and a considerable improvement over previous studies of the synchrotron emission in sky maps. We emphasise that this has not been achieved by means of a detailed parameter study of existing models but by an alternative representation - the APS - of the information contained in the sky map, and comparison with the simplest possible models, i.e. the standard GALPROP setup and simple physics describing the shells of old SNRs. This illustrates the power of the APS approach for modelling diffuse foregrounds - applications are certainly not constrained to radio and microwave frequencies alone.

As to the changes necessary for modelling the polarised emission, one needs of course to model the ordered component of the GMF (which we have neglected here), but in principle the other ingredients remain the same. In particular if we follow the picture of old SNR shells as compressions of the ISM together with the GMF, the magnetic fields should be mostly tangential which should enable easy modelling of the polarised emission from the shells. This simple picture seems to be borne out by observations of polarised emission in microwaves from the four local loops which show the polarisation vectors mostly radially orientated (see Fig. 10 of [25]). The contribution from thermal dust can be constrained from Planck observations at the highest frequencies. Furthermore, given that the (uncertain) freefree emission is unpolarised, we are confident that our approach will allow a similar (if not better) level of precision in polarisation.

Although we have stressed the validity of modelling of the APS alone, for context we also show in Fig. 11 sky maps of the different components contributing to the diffuse emission at $408 \mathrm{MHz}$. With the inclusion of turbulence up to an outer scale of $400 \mathrm{pc}$, the diffuse emission of the ISM (top left panel) looks very similar, in particular the level of "patchiness". However it appears that the emission in the Galactic plane (lower right panel) is not as peaked as is observed. (To some extent this could have been anticipated as the GALPROP model is not tuned to match radio angular profiles.) The Galactic population of SNR shells (middle left panel) does contribute mainly in the plane, since most of them are far away, and brings the latitudinal profile into agreement with the observed one. Note that there are still a couple of shells at distances $\lesssim 1 \mathrm{kpc}$ that lead to visible loops in the sky map. The most obvious difference to the sky map for the local shells, i.e. those with distances $\lesssim 500 \mathrm{pc}$ (middle left panel) is, apart from the larger angular size, their offset from the Galactic plane. (We have constrained the centres of the non-local shells in our MC simulation to lie in the Galactic plane but loosening this constraint does not change the APS much.) Finally, the sum of all components (bottom left panel) shows a remarkable resemblance to the observed sky map (bottom right panel). Note that the observed radio loops do not possess the perfect ring-like structures as assumed in our simplified approach in $\S 3.6$; the emissivity probably varies due to the non-uniform evolution of the SNR, the overall structure of the ordered GMF and the interaction and overlap of shells. However, the APS is mostly sensitive to the overall angular structure so this does not result in too big a deviation. 

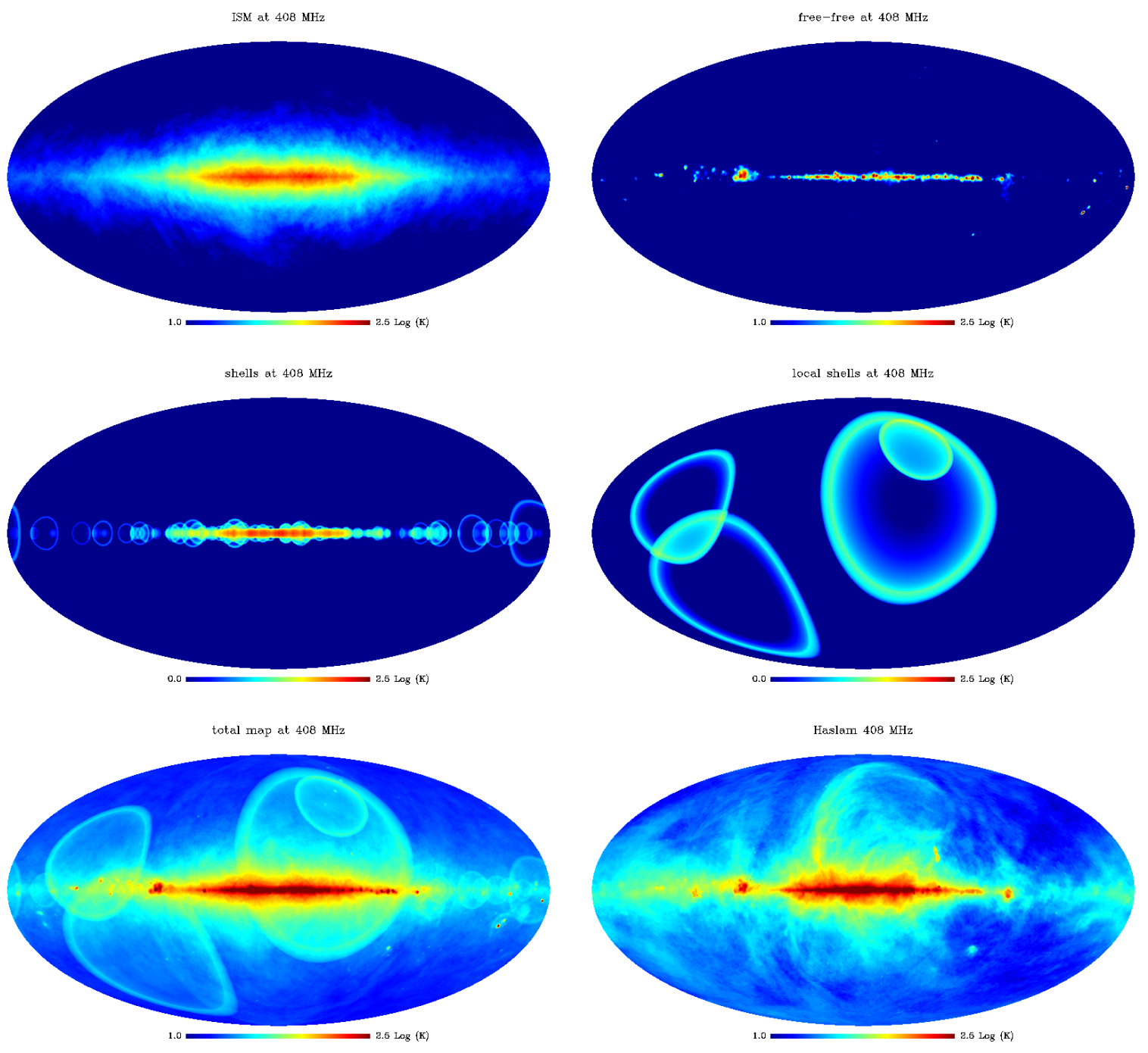

Figure 11. Top left: ISM synchrotron emission on large (modelled by GALPROP) and small scales. Top right: Free-free emission, extrapolated from the WMAP MEM free-free map. Middle left: Synchrotron emission from the Galactic population of shells of old SNRs. Middle right: Synchrotron emission from the four local shells of old SNRs, viz. Loop I - IV. Bottom left: The sum of synchrotron emission on large and small scales, free-free emission, Galactic population of shells and local shells. Bottom right: The $408 \mathrm{MHz}$ all-sky survey.

\section{Conclusions}

We have presented a new model for the diffuse, Galactic synchrotron radio background that takes into account variations of the emissivity from the scale of the Galaxy as a whole, down to the scale of interstellar magnetic turbulence. We uncovered a lack of power on intermediate scales when only the variation of the ISM emissivity is taken into account (besides free-free emission and unsubtracted point sources). We argued that this deficit is most likely cured by the inclusion of angular correlations due to the presence of $\mathcal{O}(1000)$ old SNR shells, which are similar to the known four local 'radio loops'. Even in the simplest approach, i.e. when we fix the free parameters using other observables, the agreement with observations 
at $408 \mathrm{MHz}$ is quite remarkable. This is mainly because the chosen observable, i.e. the APS, is particularly appropriate in the sense that it averages out much of the uninteresting stochasticity and highlights the dependence on the underlying physical model. We believe that the agreement can be improved even further by calibrating our model to (point source subtracted) all-sky maps at other frequencies where synchrotron emission is still dominant, e.g. at $1420 \mathrm{MHz}$ [59-61] and $2326 \mathrm{MHz}$ [62]. This will be addressed in future work..

\section{Acknowledgements}

SS thanks the Niels Bohr International Academy, Copenhagen for a Visiting Professorship. PM thanks Prof Pavel Naselsky of the 'Discovery Center', Niels Bohr Institute for support and for very useful discussions. This work was supported in part by the Department of Energy contract DE-AC02-76SF00515 and the KIPAC Kavli Fellowship made possible by The Kavli Foundation. We have benefitted from very helpful comments by the Referee.

\section{A The monopole compared to the total power}

Given a (rescaled) synchrotron skymap,

$$
J(\theta, \phi)=\int \mathrm{d} s w(s) B_{\perp}^{2}(\mathbf{r}(s, \theta, \phi)),
$$

the monopole is proportional to the squared average of this sky map:

$$
\mathcal{C}_{0}=\left|a_{00}\right|^{2}=4 \pi\left(\frac{1}{4 \pi} \int \mathrm{d} \Omega J(\theta, \phi)\right)^{2}=4 \pi \frac{2}{3}\left(\frac{\sqrt{\pi}}{2} R \int \mathrm{d}^{3} k \frac{\mathrm{d} B^{2}}{\mathrm{~d}^{3} k}\right)^{2} .
$$

The total power is, on the other hand, defined as the square of the map integrated over the whole sky,

$$
\mathcal{C}_{\text {tot }}=\int \mathrm{d} \Omega J^{*}(\theta, \phi) J(\theta, \phi)=\sum_{l}(2 l+1) \mathcal{C}_{l},
$$

i.e. the weighted sum of the APS. In the present case, i.e. for a power law turbulence spectrum

$$
\frac{\mathrm{d} B^{2}}{\mathrm{~d}^{3} k}=\mathcal{F}_{0}^{2} k^{-11 / 3} \mathrm{e}^{-k_{0}^{2} / k^{2}},
$$

it can be computed via eq. (3.6). In Fig. 13 we show $\left(\mathcal{C}_{\text {tot }}-\mathcal{C}_{0}\right) / \mathcal{C}_{0}$, i.e. the power in modes $l>0$ relative to the power in the monopole, as a function of $L / R$. This is essentially the variance of a sky map $J(\theta, \phi)$ divided by its mean square:

$$
\frac{\mathcal{C}_{\text {tot }}-\mathcal{C}_{0}}{\mathcal{C}_{0}}=\frac{\left\langle J^{2}\right\rangle-\langle J\rangle^{2}}{\langle J\rangle^{2}},
$$

where the angled brackets denote averages over the sky. It is apparent that the multipoles $l>0$ only contribute to the total power of the sky map when $L / R>1$. However, this would mean that the outer scale of turbulence is larger than the column depth - a contradiction in terms. We conclude that for realistic parameter combinations, e.g. $L \sim 100 \mathrm{pc}$ and $R>1 \mathrm{kpc}$ the monopole always dominates the total power of the map. This is essentially a consequence of the central limit theorem: as the number of turbulent eddies that is averaged over by the LoS integration becomes large, the variance around the average gets small. 


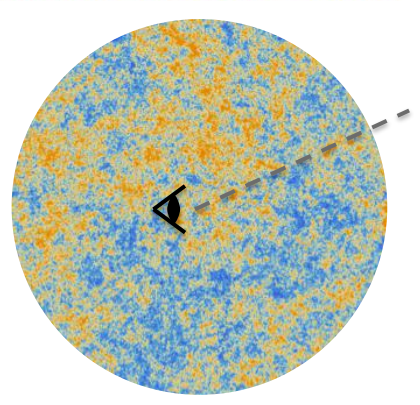

Figure 12. Observer at the centre of a turbulent sphere as in the analytical computation for small-scale varying turbulence.

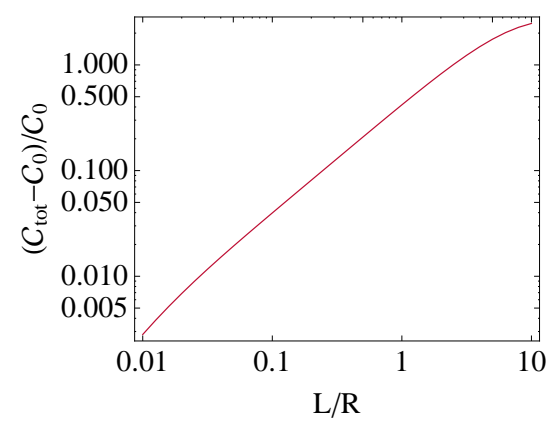

Figure 13. Power in modes $l>0$ relative to the power in the monopole, as a function of $L / R$.

\section{B Auxiliary function $J_{l}(a)$}

We start evaluating the integral $J_{l}(a)$ by partial integration and by using recurrence relations of the Legendre polynomials,

$$
\begin{aligned}
J_{l}(a) & =\int_{a}^{1} \mathrm{~d} z^{\prime} P_{l}\left(z^{\prime}\right) \sqrt{z^{2}-a^{2}} \\
& =\left[\frac{1}{2 l+1}\left(P_{l+1}(z)-P_{l-1}(z)\right) \sqrt{z^{2}-a^{2}}\right]_{a}^{1} \\
& -\frac{1}{2 l+1} \int_{a}^{1} \mathrm{~d} z \frac{z}{\sqrt{z^{2}-a^{2}}}\left(P_{l+1}(z)-P_{l-1}(z)\right) \\
& =-\frac{1}{2 l+1} \int_{a}^{1} \frac{\mathrm{d} z}{\sqrt{z^{2}-a^{2}}}\left(\frac{l+2}{2 l+3} P_{l+2}(z)+\left(\frac{l+1}{2 l+3}-\frac{l}{2 l-1}\right) P_{l}(z)-\frac{l-1}{2 l-1} P_{l-2}(z)\right) .
\end{aligned}
$$

Integrals of this form compute as the real part of the same integrand but with the lower boundary at 0 :

$$
\int_{a}^{1} \frac{\mathrm{d} z}{\sqrt{z^{2}-a^{2}}} P_{l}(z)=\Re\left\{\int_{0}^{1} \frac{\mathrm{d} z}{\sqrt{z^{2}-a^{2}}} P_{l}(z)\right\} .
$$

The integral in the brackets can now be solved by exploiting that $\mathrm{d}(\operatorname{arsinh} z) / \mathrm{d} z=1 / \sqrt{1+z^{2}}$ and expanding arsinh $z$ into a power series. The Legendre transform is evaluated separately for odd and even powers in $z$ but in both cases, we find:

$$
\frac{1}{\alpha} \int_{0}^{1} \frac{\mathrm{d} z}{\sqrt{\left(\frac{z}{\alpha}\right)^{2}+1}} P_{l}(z)=\frac{\sqrt{\pi}}{2 \alpha}{ }_{3} \tilde{F}_{2}\left(1, \frac{1}{2}, \frac{1}{2} ; 1-\frac{l}{2}, \frac{3}{2}+\frac{l}{2} ;-\frac{1}{\alpha^{2}}\right),
$$

where we have set $\alpha=i a$ and ${ }_{3} \tilde{F}_{2}$ is the regularised hypergeometric function. After some algebra, this reduces to

$$
\frac{1}{\alpha} \int_{0}^{1} \frac{\mathrm{d} z}{\sqrt{\left(\frac{z}{\alpha}\right)^{2}+1}} P_{l}(z)=\frac{(-1)^{l / 2} \alpha^{-l-1}}{2 \sqrt{\pi}}{ }_{2} \tilde{F}_{1}\left(\frac{1}{2}+\frac{l}{2}, \frac{1}{2}+\frac{l}{2} ; \frac{3}{2}+l ;-\frac{1}{\alpha^{2}}\right) .
$$


Taking into account that $\alpha$ is purely imaginary, we finally obtain the real part:

$$
\int_{a}^{1} \frac{\mathrm{d} z}{\sqrt{z^{2}-a^{2}}} P_{l}(z)=\Re\left\{\int_{0}^{1} \frac{\mathrm{d} z}{\sqrt{z^{2}-a^{2}}} P_{l}(z)\right\}=\sqrt{1-a^{2}}{ }_{2} F_{1}\left(\frac{1}{2}-\frac{l}{2}, 1+\frac{l}{2}, \frac{3}{2}, 1-a^{2}\right) .
$$

\section{References}

[1] B. Y. Mills, Nonthermal Radio Frequency Radiation from the Galaxy, Ann.Rev.Astron.Astrophys. 2 (1964) 185.

[2] W. Hu and S. Dodelson, Cosmic microwave background anisotropies, Ann.Rev.Astron.Astrophys. 40 (2002) 171, [astro-ph/0110414].

[3] WMAP Collaboration, C. Bennett et. al., First year Wilkinson Microwave Anisotropy Probe (WMAP) observations: Foreground emission, Astrophys.J.Suppl. 148 (2003) 97, [astro-ph/0302208].

[4] J. Dunkley, A. Amblard, C. Baccigalupi, M. Betoule, D. Chuss, et. al., CMBPol Mission Concept Study: Prospects for polarized foreground removal, AIP Conference Proceedings 1141 (2009) 222, [arXiv:0811.3915].

[5] COrE Collaboration, F. Bouchet et. al., COrE (Cosmic Origins Explorer) A White Paper, arXiv: 1102.2181 .

[6] J. Delabrouille, M. Betoule, J.-B. Melin, M.-A. Miville-Deschenes, J. Gonzalez-Nuevo, et. al., The pre-launch Planck Sky Model: a model of sky emission at submillimetre to centimetre wavelengths, arXiv:1207.3675.

[7] P. Mertsch and S. Sarkar, Systematic effects in the extraction of the 'WMAP haze', JCAP 1010 (2010) 019, [arXiv:1004.3056].

[8] Planck Collaboration, P. Ade et. al., Planck Intermediate Results. IX. Detection of the Galactic haze with Planck, arXiv:1208.5483.

[9] A. N. Kolmogorov, The local structure of turbulence in incompressible viscous fluid at very large Reynolds numbers, Dokl. Akad. Nauk. SSSR 30 (1941) 299.

[10] P. Goldreich and S. Sridhar, Magnetohydrodynamic turbulence revisited, Astrophys.J. 485 (1997) 680.

[11] R. Trotta, G. Johannesson, I. Moskalenko, T. Porter, R. R. de Austri, et. al., Constraints on cosmic-ray propagation models from a global Bayesian analysis, Astrophys.J. 729 (2011) 106, [arXiv: 1011.0037].

[12] D. Maurin, A. Putze, and L. Derome, Systematic uncertainties on the cosmic-ray transport parameters: Is it possible to reconcile $B / C$ data with delta $=1 / 3$ or delta $=1 / 2$ ?, Astron.Astrophys. 516 (2010) A67, [arXiv:1001.0553].

[13] R. Beck and R. Wielebinski, Magnetic fields in galaxies, vol. 5, ch. 13. Springer, 2013. arXiv: 1302.5663.

[14] A. V. Chepurnov, The galactic foreground angular spectra, Astron. Astrophys. Trans. 17:4 (1998) 281.

[15] J. Cho and A. Lazarian, MHD turbulence as a foreground for CMB studies, Astrophys.J. 575 (2002) L63, [astro-ph/0205284].

[16] J. Cho and A. Lazarian, Galactic foregrounds: Spatial fluctuations and a procedure of removal, Astrophys.J. 720 (2010) 1181-1201, [arXiv:1007.3740].

[17] A. Lazarian and D. Pogosyan, Statistical Description of Synchrotron Intensity Fluctuations: Studies of Astrophysical Magnetic Turbulence, Astrophys.J. 747 (2012) 5, [arXiv:1105.4617]. 
[18] M. Regis, Galactic Magnetic Turbulence from Radio data, Astropart. Phys. 35 (2011) 170-176, [arXiv: 1101.5524].

[19] A. Strong, E. Orlando, and T. Jaffe, The interstellar cosmic-ray electron spectrum from synchrotron radiation and direct measurements, Astron.Astrophys. 534 (2011) A54, [arXiv: 1108.4822].

[20] T. Bringmann, F. Donato, and R. A. Lineros, Radio data and synchrotron emission in consistent cosmic ray models, JCAP 1201 (2012) 049, [arXiv:1106.4821].

[21] G. Di Bernardo, C. Evoli, D. Gaggero, D. Grasso, and L. Maccione, Cosmic Ray Electrons, Positrons and the Synchrotron emission of the Galaxy: consistent analysis and implications, JCAP 1303 (2013) 036, [arXiv:1210.4546].

[22] X. Sun, W. Reich, A. Waelkens, and T. Enslin, Radio observational constraints on Galactic 3D-emission models, Astron. \& Astrophys. 477 (2008) 573, [arXiv:0711.1572].

[23] X. Sun and W. Reich, The Galactic halo magnetic field revisited, Res.Astron.Astrophys. 10 (2010) 1287, [arXiv:1010.4394].

[24] L. Fauvet, J. Macias-Perez, J. Aumont, F. Desert, T. Jaffe, et. al., Joint 3D modelling of the polarized Galactic synchrotron and thermal dust foreground diffuse emission, Astron. \& Astrophys. 526 (2011) 145, [arXiv:1003.4450].

[25] WMAP Collaboration, L. Page et. al., Three year Wilkinson Microwave Anisotropy Probe (WMAP) observations: polarization analysis, Astrophys.J.Suppl. 170 (2007) 335, [astro-ph/0603450].

[26] E. Orlando, A. Strong, I. Moskalenko, T. Porter, G. Johannesson, et. al., Cosmic-ray electrons, synchrotron and magnetic fields in the Galaxy, Proc. 31st International Cosmic Ray Conference, Lodz (2009) [arXiv:0907.0553].

[27] T. R. Jaffe, A. J. Banday, J. P. Leahy, S. Leach, and A. W. Strong, Connecting Synchrotron, Cosmic Rays, and Magnetic Fields in the Plane of the Galaxy, MNRAS 416 (2011) 1152, [arXiv: 1105.5885].

[28] T. Jaffe, K. Ferriere, A. Banday, A. Strong, E. Orlando, et. al., Comparing Polarised Synchrotron and Thermal Dust Emission in the Galactic Plane, arXiv:1302.0143.

[29] G. Badhwar, R. Daniel, and S. Stephens, Cosmic Ray Electrons and Galactic Radio Emission-a Conflict?, Nature 265 (1977) 424-426.

[30] C. F. McKee and J. P. Ostriker, A theory of the interstellar medium - Three components regulated by supernova explosions in an inhomogeneous substrate, ApJ 218 (1977) 148.

[31] H. van der Laan, Expanding supernova remnants and galactic radio sources, MNRAS 124 (1962) 125.

[32] R. Cowsik and J. Mitteldorf, Turbulence-Enhanced Synchrotron Radiation in the Galaxy, ApJ 189 (1974) 51-54.

[33] S. Sarkar, Does the galactic synchrotron radio background originate in old supernova remnants, MNRAS 199 (1982) 97-108.

[34] C. G. T. Haslam et. al., A 408 MHz all-sky continuum survey. II - The atlas of contour maps, Astron. E Astrophys. Suppl. Series 47 (1982).

[35] K. Gorski, E. Hivon, A. Banday, B. Wandelt, F. Hansen, et. al., HEALPix - A Framework for high resolution discretization, and fast analysis of data distributed on the sphere, Astrophys.J. 622 (2005) 759-771, [astro-ph/0409513].

[36] M. S. Longair, High Energy Astrophysics. Cambridge University Press, 2010.

[37] A. E. Vladimirov, S. W. Digel, G. Johannesson, P. F. Michelson, I. V. Moskalenko, et. al., 
GALPROP WebRun: an internet-based service for calculating galactic cosmic ray propagation and associated photon emissions, Comput.Phys.Commun. 182 (2011) 1156-1161,

[arXiv: 1008.3642].

[38] A. Strong, T. Porter, S. Digel, G. Johannesson, P. Martin, et. al., Global cosmic-ray related luminosity and energy budget of the Milky Way, Astrophys.J. 722 (2010) L58-L63, [arXiv: 1008.4330].

[39] R. Beck, A. Shukurov, D. Sokoloff, and R. Wielebinski, Systematic bias in interstellar magnetic field estimates, Astron.Astrophys. 411 (2003) [astro-ph/0307330].

[40] T. Jaffe, J. Leahy, A. Banday, S. Leach, S. Lowe, et. al., Modelling the Galactic Magnetic Field on the Plane in 2D, MNRAS 401 (2009) 1013, [arXiv:0907.3994].

[41] M. Kachelriess and S. Ostapchenko, Deriving the cosmic ray spectrum from gamma-ray observations, Phys.Rev. D86 (2012) 043004, [arXiv:1206.4705].

[42] V. Ptuskin, I. V. Moskalenko, F. Jones, A. Strong, and V. Zirakashvili, Dissipation of magnetohydrodynamic waves on energetic particles: impact on interstellar turbulence and cosmic ray transport, Astrophys.J. 642 (2006) 902-916, [astro-ph/0510335].

[43] P. Blasi, E. Amato, and P. D. Serpico, Spectral breaks as a signature of cosmic ray induced turbulence in the Galaxy, Phys.Rev.Lett. 109 (2012) 061101, [arXiv: 1207.3706].

[44] M. Ahlers, P. Mertsch, and S. Sarkar, On cosmic ray acceleration in supernova remnants and the FERMI/PAMELA data, Phys.Rev. D80 (2009) 123017, [arXiv:0909.4060].

[45] D. R. Lorimer, The Galactic population and birth rate of radio pulsars, astro-ph/0308501.

[46] V. Ptuskin, Propagation of galactic cosmic rays, Astropart. Phys. 39-40 (2012) 44-51.

[47] J. Giacalone and J. R. Jokipii, The Transport of Cosmic Rays across a Turbulent Magnetic Field, ApJ 520 (1999) 204-214.

[48] C. Dickinson, R. Davies, and R. Davis, Towards a free-free template for CMB foregrounds, MNRAS 341 (2003) 369, [astro-ph/0302024].

[49] WMAP Collaboration, B. Gold et. al., Five-Year Wilkinson Microwave Anisotropy Probe (WMAP) Observations: Galactic Foreground Emission, Astrophys.J.Suppl. 180 (2009) 265-282, [arXiv:0803.0715].

[50] L. La Porta, C. Burigana, W. Reich, and P. Reich, The Impact of Galactic synchrotron emission on $C M B$ anisotropy measurements. I. Angular power spectrum analysis of total intensity all-sky surveys, Astron. 6 Astrophys. 479 (2008) 641, [arXiv:0801.0547].

[51] E. M. Berkhuijsen, C. G. T. Haslam, and C. J. Salter, Are the galactic loops supernova remnants?, A\&A 14 (1971) 252.

[52] C. J. Salter, Loop-I the North Polar Spur - a Major Feature of the Local Interstellar Environment, Bulletin of the Astronomical Society of India 11 (1983) 1.

[53] M. Wolleben, A New Model For The Loop-I (The North Polar Spur) Region, Astrophys.J. 664 (2007) 349-356, [arXiv:0704.0276].

[54] M. Pohl, A. A. Abdo, A. Atoyan, M. G. Baring, J. F. Beacom, et. al., Section on Supernova remnants and cosmic rays of the White Paper on the Status and Future of Ground-based Gamma-ray Astronomy, arXiv:0810.0673.

[55] C. McKee and J. Ostriker, A theory of the interstellar medium - Three components regulated by supernova explosions in an inhomogeneous substrate, Astrophys.J. 218 (1977) 148.

[56] R. Bandiera and O. Petruk, Analytic solutions for the evolution of radiative supernova remnants, Astron.Astrophys. 419 (2004) 419, [astro-ph/0402598].

[57] B. Gold, N. Odegard, J. Weiland, R. Hill, A. Kogut, et. al., Seven-Year Wilkinson Microwave 
Anisotropy Probe (WMAP) Observations: Galactic Foreground Emission, Astrophys.J.Suppl. 192 (2011) 15, [arXiv: 1001.4555].

[58] C. Bennett, D. Larson, J. Weiland, N. Jarosik, G. Hinshaw, et. al., Nine-Year Wilkinson Microwave Anisotropy Probe (WMAP) Observations: Final Maps and Results, arXiv: 1212.5225.

[59] W. Reich, A radio continuum survey of the northern sky at 1420 MHz. I, A\&AS 48 (1982) 219.

[60] P. Reich and W. Reich, A radio continuum survey of the northern sky at 1420 MHz. II, A\&AS 63 (1986) 205.

[61] P. Reich, J. C. Testori, and W. Reich, A radio continuum survey of the southern sky at 1420 $M H z$. The atlas of contour maps, A\&A 376 (2001) 861.

[62] J. L. Jonas, E. E. Baart, and G. D. Nicolson, The Rhodes/HartRAO 2326-MHz radio continuum survey, MNRAS 297 (1998) 977-989. 